\title{
Thermal stresses in hybrid materials with auxetic inclusions
}

\author{
Elena Pasternak ${ }^{\mathrm{a}, 2}$, Igor Shufrin ${ }^{\mathrm{a}, \mathrm{b}, 1}$ and Arcady V. Dyskin ${ }^{\mathrm{b}, 3}$ \\ ${ }^{a}$ School of Mechanical and Chemical Engineering, The University of Western Australia, 35 \\ Stirling Highway, Crawley WA 6009, Australia \\ ${ }^{\mathrm{b}}$ School of Civil, Environmental and Mining Engineering, The University of Western \\ Australia, 35 Stirling Highway, Crawley WA 6009, Australia \\ ${ }^{1}$ Corresponding author: e-mail igor.shufrin@uwa.edu.au \\ 2 e-mail: elena.pasternak@uwa.edu.au \\ 3 e-mail: arcady.dyskin@uwa.edu.au
}

\begin{abstract}
We study thermo-elastic behaviour of the hybrid materials with auxetic inclusions. To this end we analyse representative volume elements consisting of a large number of randomly positioned cubic inclusions using the finite element method and determine the effective coefficient of thermal expansion and the average thermal stresses in constrained hybrid materials developed as a result of a uniform temperature change. We study the effect of Poisson's ratio, Young's modulus and the coefficient of thermal expansions of the inclusions on the effective coefficient of thermal expansion and thermal stresses. We demonstrate that the auxeticity of the inclusions can reduce thermal stresses, which is controlled by the values of the Poisson's ratio and the coefficients of thermal expansion of the inclusions and matrix.
\end{abstract}

Keywords: Thermal stresses, Auxetic inclusions, Effective characteristics, Coefficient of thermal expansion 


\section{Introduction}

Particle reinforced composites are a common form of hybrid materials used in engineering structures. The particles are added to a matrix to alter the overall properties of the hybrid in terms of mechanical moduli, strength, electric and thermal conductivity and etc. [1] and thus improve performance and enhance functionality. In general, these effective properties depend upon the properties of both matrix and inclusions, concentration of the inclusions and their shape. Recently we reported on a new class of hybrid materials consisting of auxetic (possessing negative Poisson's ratio (NPR)) and non-auxetic phases [2]. We demonstrated that by adding auxetic inclusions in a non-auxetic matrix one could achieve a considerable increase in stiffness over the stiffness of the matrix and inclusions. We studied the influence of various parameters on the mechanical performance of these hybrids. We showed that the stiffening effect is controlled by the values of the Poisson's ratio of the matrix and inclusions, shape of the auxetic inclusions and their concentration [2]. In the present study, we consider the thermal deformation of the hybrid materials with auxetic inclusions. We evaluate the coefficient of thermal expansion (CTE) and thermal stresses occurring in these hybrids.

Thermal deformations and thermally induced stresses play a critical role in many engineering applications ranging from microelectronic and optic devices [3] to gas turbines and jet engines $[4,5]$. The thermal stresses are a common source of mechanical failures, such as brittle fracture, excessive deformations, thermal fatigue, material degradation and buckling. In addition, the thermal distortions cause functional failures in systems that are sensitive to large deformations, such as high precision optics and electronics [3]. One of the ways to manage the thermo-mechanical design is to employ materials with enhanced thermal properties [4] or in multipart cases with matching thermal properties [6]. In this sense, the hybrid material approach as introduced by Ashby and Brechet [1] offers a possibility to create the materials optimally tuned to the purpose. The most important feature of this concept is that the internal architecture and scale are introduced as design parameters. In particular, the separation of scales allows the design of components with unusual properties, where the effective properties of the components are first defined by averaging over suitable representative volume elements at the component scale and then the effective characteristics of the hybrid are designed at the upper scale based on these properties (e.g. [2]).

Following this approach the control of the thermal properties of a multiphase composite can be carried out in two ways: (i) by designing the microstructure of the hybrid that would 
exhibit the desired thermal expansion behaviour at the macroscale level or (ii) by altering the effective thermal and mechanical properties of the phases. The first approach has been widely used to design hybrid microstructures leading to the negative thermal expansion (NTE) using periodical lattices, cellular structures and kinematic actuators. The NTE effect in these microstructures was achieved through various bimaterial elements at the microscale (the same scale as the microstructure). One of the popular designs was a lattice consisting of bimaterial cells composed of a lower CTE stiff frame and a higher CTE soft core [7-12]. A range of the lattices with low or negative CTE that was developed capitalising on this concept includes rigidly connected curved hexagonal honeycombs [8], rigidly connected cells with straight ribs [9, 10, 12], non-identical cells [11] and thin films [12]. In models of the cellular microstructures with tunable CTE, the biomaterial approach was applied to design the cell ribs. This included the cells with curved bimaterial ribs [13], and 2D and 3D lattices composed of nested biomaterial tubes $[14,15]$. The periodic frame structures consisting of rods with altering individual CTEs were also shown to exhibit the NTE effect [16, 17]. A systematic approach to design of NTE microstructures based on topology optimisation of the microstructural architecture using a library of shapes so-called Freedom, Actuation, And Constraint Topologies (FACT) was explored in [18].

The second approach to the design of multiphase composites with tunable thermal properties capitalises on the concept of particle-reinforced composites. In this case, the control over the thermal performance of the hybrid is achieved by introducing the inclusions of different shapes with special thermal and mechanical properties $[19,20]$. The discovery of tungstate and molybdate compounds $[21,22]$ exhibiting isotropic NTE inspired the use of these materials for improving the thermal performance of composites [19, 20, 23, 24]. At the same time, the use of other materials was also investigated including negative stiffness phase [25] and negative Poisson's ratio [26, 27]. The shapes and arrangement of the phases were also considered. Hard disc, cylindrical and needle shapes inclusions with high positive CTE embedded into a soft matrix with low positive CTE were shown exhibiting the NTE under certain conditions [28]. Laminated stacks with alternating auxetic and non-auxetic layers with negative and positive CTE can be designed to provide low or even negative CTE [29].

Here we consider the application of auxetic inclusions with negative and positive CTE. The auxetic materials have attracted vast research attention during last two decades. A range of auxetic systems that have been identified and studied included polymers and foams (e.g. [3033]), molecular models [34-38], auxetic crystals (e.g. [39-41]), nanoauxetics [42-44], 
composites (e.g. [45-51]), auxetic lattices [52, 53], hollow sphere stacks [54] and auxetic metamaterials (e.g. [55-59]). The studies on the auxetic behaviour demonstrated many beneficial properties associated with the auxetic behaviour, such as improved shear modulus, enhanced fatigue, indentation and impact resistance [60-62]. It was also shown that in some cases the auxetic behaviour reduces the stress created due to the thermal expansion [27]. The main objective of this paper is to study the influence of the auxetic phase on the thermal stresses in hybrid materials.

This paper is structured as follows. First, we introduce a representative volume element with randomly distributed cubic auxetic inclusions $[2,63]$ and analyse it using the finite element method. Then we compare the obtained effective CTEs with the existing methods for evaluation of effective characteristics of two-phase composites. Finally, we investigate the thermal stress levels in the hybrids with auxetic inclusions with positive and negative CTE and various Young's moduli and negative Poisson's ratio.

\section{Thermo-elastic behaviour of hybrid materials with auxetic inclusions. Finite element model}

Consider a hybrid consisting of an isotropic matrix with conventional material properties (positive Poisson's ratio and positive thermal expansion coefficient) and auxetic inclusions. We assume that the auxetic inclusions have cubic shape and may exhibit both positive and negative thermal expansion. We also assume that the separation of scales exists implying that one can introduce a volume element (a representative volume element) with the dimensions that are much larger than the sizes of inclusions and distances between them, while still much smaller than the characteristic length of variations of the externally applied fields. Then the effective characteristics of the hybrid (e.g. effective moduli, effective coefficient of thermal expansion) can be calculated through the fields averaged over the volume element. To evaluate the continuum properties of the hybrid we model a representative volume element (RVE) of a volume $V$ by the finite element method as shown in Fig 1. The RVE is divided into a regular cubic grid, in which each cube (depicted by a solid line in Fig 1) represents either the inclusion or a part of the matrix. Each inclusion is meshed using 3D finite elements that are also regularly arranged into the cubic grid plotted by dash lines in Fig 1. It is also assumed that the bond between the inclusions and the matrix is rigid. The auxetic inclusions are randomly distributed and allowed to form clusters of complex geometry typical at high 
volumetric fractions of the inclusions. A particular computational realisation of the hybrid is achieved by randomly assigning the material properties to the cubic elements in the grid. As all blocks have the same dimensions, the concentration of auxetic inclusions is defined as the ratio of the number of the blocks assigned the negative Poisson's ratio to the total number of the blocks. The negative and positive Poisson's ratio blocks are shuffled using the random permutation function incorporated in MATLAB. This function uses the Mersenne-Twister pseudo-random number generator producing a uniform distribution over the total number of blocks [64].

We study the thermo-elastic behaviour of the hybrid by considering two cases: the free thermal expansion test for evaluation of the effective CTE and the confined thermal loading test for the evaluation of the thermal stresses. The computational models for the simulation of these conditions are outlined next.

\subsection{Evaluation of the effective coefficient of thermal expansion}

To determine the effective CTE we apply a uniform change of temperature $\Delta T$ to the unconstrained volume of the hybrid. We assume that the RVE is symmetric in all three directions and analyse only an eighth of the cubic RVE, Fig 2 . Thus, the following symmetry conditions are imposed at the planes of symmetry:

$$
\begin{aligned}
& u_{x}=\tau_{x y}=\left.\tau_{x z}\right|_{x=0}=0 \\
& u_{y}=\tau_{y x}=\left.\tau_{y z}\right|_{y=0}=0 \\
& u_{z}=\tau_{z x}=\left.\tau_{z y}\right|_{z=0}=0
\end{aligned}
$$

where $u_{x}, u_{y}, u_{z}$ are the displacements in the direction of the coordinate axes and $\tau_{i j}$ are the shear stresses. At the external faces the stress free boundary conditions are specified as follows:

$$
\begin{aligned}
& \sigma_{x x}=\tau_{x y}=\left.\tau_{x z}\right|_{x=L}=0 \\
& \sigma_{y y}=\tau_{y x}=\left.\tau_{y z}\right|_{y=L}=0 \\
& \sigma_{z z}=\tau_{z x}=\left.\tau_{z y}\right|_{z=L}=0
\end{aligned}
$$

The effective CTE is calculated based on the deformations caused by the thermal expansion as follows. For each concentration of the auxetic inclusions, we generate 10 random realisations for the inclusion distribution and carry out the FE modelling. Based on the 
obtained solution, we evaluate the mean normal displacement at all free faces of the RVE for each distribution and calculate the average thermal strain in each RVE dimension by dividing the displacement with the respective RVE size. As we assumed the symmetric distribution of deformation, the same normal displacement appears at all free faces and therefore the average thermal strain is calculated with respect to the half size, $L$ of the RVE, Fig 2 . The averaging over the random realisations yields the effective linear thermal expansion in each direction. The effective linear CTE is obtained by averaging the effective strains in all three directions as

$\alpha_{\text {eff }}=\left\langle\frac{\varepsilon_{x, e f f}}{\Delta T}, \frac{\varepsilon_{y, e f f}}{\Delta T}, \frac{\varepsilon_{z, e f f}}{\Delta T}\right\rangle$

where $\Delta T$ is the change of temperature and $\varepsilon_{x, e f f}, \varepsilon_{y, e f f}$ and $\varepsilon_{z, e f f}$ are the linear thermal strains in the $x, y$ and $z$ directions respectively.

\subsection{Evaluation of thermal stresses}

To evaluate the thermal stresses, we apply the thermal loading to the confined volume element of the hybrid. The RVE geometry and boundary conditions are shown in Fig 3. Also here we consider only one eighth of the volume and adopt the symmetry conditions (1). The external faces are constrained in the respective normal directions by the following boundary conditions:

$$
\begin{aligned}
& u_{x}=\tau_{x y}=\left.\tau_{x z}\right|_{x=L}=0 \\
& u_{y}=\tau_{y x}=\left.\tau_{y z}\right|_{y=L}=0 \\
& u_{z}=\tau_{z x}=\left.\tau_{z y}\right|_{z=L}=0
\end{aligned}
$$

The resulting magnitude of stress developing at each RVE face is evaluated for each distribution of the auxetic inclusions. We again use 10 random realisations for each level of the inclusion concentration. The constraining reaction forces are calculated for each realisation at three external faces of the RVE through the FE analysis. The normal stress is then defined as the obtained reactions over the corresponding area of the RVE face and averaged for all realisations. Note that here we also calculate the normal stresses as applied over a fourth of the face area, $L^{2}$, Fig 3 . 


\subsection{Thermal expansion coefficient in two-phase composites}

In order to verify the computational model presented above, firstly we compare the results with the existing models for prediction of CTE in two-phase composites. This topic has been a subject of many research studies. The constitutive response of two-phase composites subjected to a temperature change does not obey simple mixture rules [65-70], and therefore requires special considerations. A thorough survey of the models for the evaluation of the CTE was presented in [71]. A detailed insight into thermal expansion behaviour of composites can also be found in [72].

One of the first formulas for prediction of the CTE in isotropic two-phase composites with arbitrary microstructure was proposed by Turner [65]:

$\alpha_{e f f}=\frac{\alpha_{1} V_{1} K_{1}+\alpha_{2} V_{2} K_{2}}{V_{1} K_{1}+V_{2} K_{2}}$

where $\alpha_{\text {eff }}$ is the effective coefficient of thermal expansion, $K_{1}$ and $K_{2}$ are the bulk moduli, $\alpha_{1}$ and $\alpha_{2}$ are the CTEs, $V_{1}$ and $V_{2}$ are the volume fractions of the phases. It was assumed that the rate of the thermal expansions is the same for both constituents and that the shear deformation is negligible. This formula is equivalent to the Voight bound in the mechanics of composites [71] and due to its simplicity the most commonly referenced estimate for the effective CTE.

Levin [66] and Rosen and Hashin [68] derived the following formula for statistically isotropic composites based on the principle of virtual work:

$$
\alpha_{e f f}=\alpha_{1}+\frac{\alpha_{2}-\alpha_{1}}{1 / K_{2}-1 / K_{1}}\left(\frac{1}{K_{e f f}}-\frac{1}{K_{1}}\right)
$$

where $K_{\text {eff }}$ is the effective bulk moduli. This expression allows for evaluation of the upper and low bounds for $\alpha_{\text {eff }}$ by introducing into Eq.(6) the Hashin-Shtrikman bounds for $K_{\text {eff }}$ [73].

$$
\begin{aligned}
& K_{-}=K_{1}+\frac{V_{2}}{\frac{1}{K_{2}-K_{1}}+\frac{3 V_{1}}{3 K_{1}+4 G_{1}}} \\
& K_{+}=K_{2}+\frac{V_{1}}{\frac{1}{K_{1}-K_{2}}+\frac{3 V_{2}}{3 K_{2}+4 G_{2}}}
\end{aligned}
$$


where $G_{1}$ and $G_{2}$ are the shear moduli of the phases.

It can be observed from Eqs. (5) and (6) that the effective CTE of the hybrid will only differ from the CTE of the phases if the CTE of the components are also different. At the same time, the thermo-elastic behaviour of the hybrid with phases exhibiting dissimilar CTE will be affected by the Poisson's ratio manifesting itself through the variation in the bulk modulus.

\section{Results and Discussion}

In this section, we present the quantitative study on thermal behaviour of hybrids with auxetic inclusions. In order to evaluate the effective CTE and the levels of developed thermal stresses, the computational models described in Sections 2.1 and 2.2 are implemented in ABAQUS Standard, a commercial package for the finite element analysis. The cubic grid of the matrix and inclusion elements (see Fig 1) is built using linear 8-node brick elements (C3D8R) incorporated in ABAQUS. The parameters of the RVE are selected based on the outcomes of the finite element mesh convergence study and the study on the macroscopic uniformity of effective properties presented in [2]. Accordingly, the eighth part of the cubic RVE is modelled by a grid of $12 \times 12 \times 12$ blocks with 8 elements per each block.

The following symbolism is used to present the results. Subscripts of ' $i n$ ' and ' $m$ ' designate the properties of the inclusions and matrix respectively. We assume linear elastic behaviour for the hybrid constituents, which allows for a dimensionless parametric study, where all controlling parameters are represented by dimensionless ratios, such as $E_{i n} / E_{m}$ and $\alpha_{i n} / \alpha_{m}$. The Poisson's ratio of the matrix is taken as 0.45 throughout all examples unless otherwise specified, while the Poisson's ratios of the inclusions vary from -0.99 to 0.45 .

\subsection{Verification of the numerical model}

As discussed in Section 2.3, firstly we compare the results obtained using the presented models with the methods available in the literature. Fig 4 presents the normalised effective CTE for the hybrid with inclusions possessing high NPR of -0.9 and negative CTE calculated using different methods. All models show that the positive CTE of the matrix reduces to the negative values with an increase in the concentration of the inclusions. The results of the free expansion test (Section 2.1) are in the range of the Rosen-Hashin bounds (7) and (8) and exhibiting behaviour close to the upper bound (8). The low Rosen-Hashin bound (7) 
however, suggests much steeper decrease in the effective CTE, while the Turner's formula (5) provides results above the Rosen-Hashin's upper bound (8). To clarify this discrepancy, we calculate the effective bulk modulus of the hybrid, $K_{\text {eff }}$ and substitute it directly into Eq. (6).

To evaluate the bulk modulus of the hybrid, we use a procedure similar to the one described in Section 2, and detailed in Appendix A. The obtained values of the bulk modulus of the hybrid with the inclusions possessing NPR of -0.9 are plotted in Fig 5 together with the corresponding values of the effective Young's modulus which are taken from our previous calculations [2]. These curves demonstrate the stiffening effect of the auxetic inclusions reported previously $[2,27]$. It can also be observed that the bulk modulus is rapidly decreasing and this decrease is not coupled to the stiffening effect. This effective bulk modulus is used to calculate the effective CTE, which is also included into Fig 4. It is seen that the free expansion test is in very good agreement with the (effective) Rosen-Hashin's formula.

Next we compare the effective CTEs predicted using the presented models with the experimental results available in the literature. Table 1 presents the results for a composite consisting of a positive CTE polymer matrix (cyanate ester resin) and NTE zirconium tungstate filler whose properties were experimentally investigated in [23]. The properties of the phases are taken from reference [23] as $v_{m}=0.33, E_{m}=2.078 \mathrm{GPa}, \alpha_{m}=56.5 \times 10^{-6} \mathrm{~K}^{-1}$, $v_{i n}=0.302, E_{i n}=88.3 \mathrm{GPa}, \alpha_{i n}=-8.7 \times 10^{-6} \mathrm{~K}^{-1}$. It is seen that the FEM results are in the excellent agreement with the experimental study. Also here we observe the thermal expansion behaviour close to the Rosen-Hashin's upper bound. Table 2 shows the results for another epoxy/zirconium tungstate composite reported in [19] with the following parameters $v_{m}=0.3$, $E_{m}=1.8 \mathrm{GPa}, \alpha_{m}=200 \times 10^{-6} \mathrm{~K}^{-1}, v_{i n}=0.3, E_{i n}=88 \mathrm{GPa}, \alpha_{i n}=-8.6 \times 10^{-6} \mathrm{~K}^{-1}$. It can be seen that the experimental results are higher than the Rosen-Hashin's upper bound and the predictions of the FE analysis. The experimental results obtained in [19] for different kinds of surface coatings of the filler particles are not consistent for different filler volume fractions, e.g. in the case of $20 \%$ filler content, the silane and carboxylic acid surface treatments do not reduce the effective CTE, in the case of 30\%, the silane coating increases the CTE and carboxylic acid treatment reduces it, while in the case of $40 \%$ both treatments reduce the thermal expansion. This apparent lack of consistency in the experimental data calls for a finite element analysis with chemical coupling 


\subsection{Thermal expansion of the hybrid materials with auxetic inclusions.}

To further study the influence of the auxetic inclusions on the thermal expansion we analyse the hybrids with inclusions of various negative Poisson's ratios, CTE and Young's moduli. Fig 6 and Fig 7 present the effect of the Poisson's ratio of the inclusions with negative and positive CTE respectively on the effective thermal expansion. The ratios between the CTE of the inclusions to the matrix CTE are taken as $\alpha_{i n} / \alpha_{m}=-10$ and $\alpha_{i n} / \alpha_{m}=10$ for the negative and positive CTE respectively. It is seen that the introduction of the negative CTE inclusions reduces the effective $\mathrm{CTE}$ of the hybrid and brings it to the negative zone. However, the increase in the auxeticity of the inclusions diminishes this effect: the concentration of inclusions required for shifting the whole hybrid into the negative CTE zone increases from $24.3 \%$ for $v_{i n}=-0.4$ to $37.4 \%$ for $v_{i n}=-0.8$ and $67.5 \%$ for the extreme value of $v_{i n}=-0.99$ (see Fig 6).

Similarly considering the auxetic inclusions with higher positive CTE, we can observe the increase in the effective CTE of the hybrid (see Fig 7). It is seen that the inclusions with higher NPR values considerably reduce the rate of the CTE increase unless their concentration is close to $100 \%$. The hybrids with $v_{i n}=-0.8,-0.9$ and -0.99 require at least $70 \%$ inclusion concentration to reach $30 \%$ of the overall change in the effective CTE. It can also be observed that when Poisson's ratios of the inclusions and the matrix coincide, the effective CTE of the hybrid obeys the simple mixture rule with the values directly proportional to the inclusion concentration. This relation departs from linearity when the Poisson's ratios of the inclusions decrease towards the negative zone.

Next, we investigate the effect of the Young's modulus of the inclusions on the effective CTE of the hybrid. Since both mechanisms of auxeticity and negative thermal expansion are characterised by a relatively low Young's modulus we focus here on the inclusions with the Young's modulus lower than the one of the matrix. The effective CTEs for the hybrids with the highly auxetic inclusions of -0.99 NPR and low positive CTE are depicted in Fig 8. As before we observe that the highly auxetic inclusions reduce the rate of decrease in the effective CTE when the Young's modulus ratio $E_{i n} / E_{m}=1$. Only when the concentration of the inclusions is near $100 \%$ the reduction diminishes. The low CTE of the inclusions has almost no effect on the effective CTE of the hybrid when this stiffness ratio decreases. 


\subsection{Thermal stresses in hybrids with auxetic inclusions}

In order to study the effect of auxetic inclusions on the thermal stress in the hybrid, we normalise the average stress evaluated as described in Section 2.2 by the thermal stress developing in the matrix with no inclusions. In the constrained isotropic matrix with the bulk modulus $K_{m}$, the normal stress in any direction due to the uniform temperature change $\Delta T$ reads

$$
\sigma_{x m}=-3 \alpha_{m} \Delta T K_{m}
$$

Fig 9 presents the average stress in the hybrid with inclusions of various Poisson's ratios and a negative CTE. It is seen that when the concentration of inclusions increases the compressive stress (marked by the positive values of ratio $\sigma_{x} / \sigma_{x m}$ ) developing in the matrix gradually decreases and becomes tensile due to the negative thermal expansion effect. This figure also reveals that positive Poisson's ratio inclusions with the negative CTE provide better reduction of the thermal stresses, in particular when the Poisson's ratio approaches its upper limit of 0.5 (Fig 9b). This agrees with the decrease in the rate of reduction of the effective CTE that auxeticity of the inclusions cause, as we observed previously in Fig 6. The influence of the Poisson's ratio of inclusions on the thermal stress reduction however does not happen when we use auxetic inclusions with a positive CTE that is smaller than the one of the matrix $\left(\alpha_{i n} / \alpha_{m}=0.1\right)$. Fig 10 demonstrates that the average stress decreases with the increase in the concentration of the inclusions, while the Poisson's ratio of the inclusions in a range between $-0.7 \ldots 0$ has almost no influence on the results and becomes noticeable only for the extreme values of -0.99 and 0.9 . On the other hand, a positive Poisson's ratio of the inclusions considerably diminishes this effect. For example, the ratio $\sigma_{x} / \sigma_{x m}$ at the concentration of $50 \%$ increases from 0.2 for $v_{i n}=0$ to 0.91 for $v_{i n}=0.49$ (Fig 10b).

This trend changes again when the auxetic inclusions have a positive CTE that is higher than that of the matrix as shown in Fig 11a. It is seen that the auxeticity of inclusions leads to the reduction in the average thermal stresses even with $\alpha_{i n} / \alpha_{m}=10$ while a stronger reduction is provided by the inclusions with higher NPR. Note the limiting case of $v_{i n}=-0.99$ when the concentrations of the inclusions up to $80 \%$ do not yield the best reduction effect. The positive Poisson's ratio inclusions lead to the increase in the thermal stresses in this case with an extreme upsurge for the upper bound values (Fig 11b). 
Fig 12 presents variations of the thermal stresses in the hybrids with auxetic inclusions with the ratio of CTE $\alpha_{i n} / \alpha_{m}$ ranging from -10 to 10. It is seen that the reduction in the thermal stress can be observed across the whole range of CTE values of auxetic inclusions.

The influence of the inclusions' Young's modulus on the stress level is studied in Fig 13 using the inclusions of the extreme NPR of -0.99 and the ratio of CTE $\alpha_{i n} / \alpha_{m}=0.1$. It is seen that the softer inclusions provide better reduction in the average thermal stress.

\section{Conclusions}

In this paper, we have studied the thermo-mechanical behaviour of hybrid materials with cubic auxetic inclusions using the finite element method to determine the effective coefficients of thermal expansion (CTE) and the magnitude of average stress developing in the constrained hybrid due to the temperature change. The study was based on considering representative volume elements consisting of a large number of randomly placed inclusions. The computational models were verified through comparisons of the obtained effective CTE with models available in literature. The findings of this study can be summarised as follows:

- Introduction of the auxetic inclusions with positive CTE in the conventional matrix with positive Poisson's ratio can reduce the thermal stress developing in the hybrid.

- In the hybrids with auxetic inclusions with negative CTE, the negative CTE inclusions reduce the effective CTE of the hybrid and the average thermal stresses. However, the negative Poisson's ratio of the inclusions weakens both effects.

- In the hybrids with auxetic inclusions with positive CTE that is smaller than the CTE of the matrix, the negative Poisson's ratio has little influence on both the thermal expansion and stresses.

- In the hybrids with the auxetic inclusions with positive CTE that is higher than the CTE of the matrix, the auxeticity becomes the dominant effect leading to the reduction of both effective CTE and thermal stresses.

- The decrease in Young's modulus of the auxetic inclusions can aid the thermal stress reduction.

\section{Acknowledgements}

The authors acknowledge the financial support through ARC Discovery Grant DP120102434. 


\section{References}

[1] Ashby MF, Bréchet YJM. Designing hybrid materials. Acta Mater. 2003;51:5801-21.

[2] Shufrin I, Pasternak E, Dyskin AV. Hybrid materials with negative Poisson's ratio inclusions. Int. J. Eng. Sci. 2015;89:100-20.

[3] Suhir E. Predictive Analytical Thermal Stress Modeling in Electronics and Photonics. Appl. Mech. Rev. 2009;62:040801.

[4] Bunker RS. Gas Turbine Heat Transfer: Ten Remaining Hot Gas Path Challenges. J. Turbomach. 2006;129:193-201.

[5] Choi W, Fujiyama K, Kim B, Song G. Development of thermal stress concentration factors for life assessment of turbine casings. Int. J. Press. Vessels Pip. 2012;98:1-7.

[6] Suhir E. Modeling of the Mechanical Behavior of Materials in "High-Tech" Systems: Attributes and Review. J. Electron. Packag. 1999;121:213-21.

[7] Steeves CA, dos Santos e Lucato SL, He M, Antinucci E, Hutchinson JW, Evans AG. Concepts for structurally robust materials that combine low thermal expansion with high stiffness. J. Mech. Phys. Solids. 2007;55:1803-22.

[8] Jefferson G, Parthasarathy TA, Kerans RJ. Tailorable thermal expansion hybrid structures. Int. J. Solids Struct. 2009;46:2372-87.

[9] Berger J, Mercer C, McMeeking RM, Evans AG. The Design of Bonded Bimaterial Lattices that Combine Low Thermal Expansion with High Stiffness. J. Am. Ceram. Soc. 2011;94:s42-s54.

[10] Rhein R, Novak M, Levi C, Pollock T. Bimetallic low thermal-expansion panels of Cobase and silicide-coated $\mathrm{Nb}$-base alloys for high-temperature structural applications. Mater. Sci. Eng., A. 2011;528:3973-80.

[11] Toropova M, Steeves C. Bimaterial lattices with anisotropic thermal expansion. J. Mech. Mater. Struct. 2014;9:227-44.

[12] Yamamoto N, Gdoutos E, Toda R, White V, Manohara H, Daraio C. Thin Films with Ultra-low Thermal Expansion. Adv. Mater. 2014;26:3076-80.

[13] Lakes R. Cellular solids with tunable positive or negative thermal expansion of unbounded magnitude. Appl. Phys. Lett. 2007;90:221905.

[14] Lehman J, Lakes R. Stiff, strong zero thermal expansion lattices via the Poisson effect. J. Mater. Res. 2013;28:2499-508.

[15] Lehman J, Lakes RS. Stiff, strong, zero thermal expansion lattices via material hierarchy. Compos. Struct. 2014;107:654-63.

[16] Grima JN, Farrugia PS, Gatt R, Zammit V. A system with adjustable positive or negative thermal expansion. Proceedings of the Royal Society of London A: Mathematical, Physical and Engineering Sciences. 2007;463:1585-96.

[17] Lim T-C. Negative thermal expansion structures constructed from positive thermal expansion trusses. J. Mater. Sci. 2012;47:368-73.

[18] Hopkins JB, Lange KJ, Spadaccini CM. Designing Microstructural Architectures With Thermally Actuated Properties Using Freedom, Actuation, and Constraint Topologies. J. Mech. Des. 2013;135:061004.

[19] Miller W, Smith CW, Dooling P, Burgess AN, Evans KE. Tailored thermal expansivity in particulate composites for thermal stress management. Phys Status Solidi B.

2008;245:552-6. 
[20] Miller W, Smith CW, Dooling P, Burgess AN, Evans KE. Reduced thermal stress in composites via negative thermal expansion particulate fillers. Compos. Sci. Technol. 2010;70:318-27.

[21] Mary TA, Evans JSO, Vogt T, Sleight AW. Negative Thermal Expansion from 0.3 to 1050 Kelvin in ZrW2O8. Science. 1996;272:90-2.

[22] Evans JSO, Mary TA, Sleight AW. Negative thermal expansion materials. Physica B. 1998;241-243:311-6.

[23] Badrinarayanan P, Kessler MR. Zirconium tungstate/cyanate ester nanocomposites with tailored thermal expansivity. Compos. Sci. Technol. 2011;71:1385-91.

[24] Neely L, Kochergin V, See E, Robinson H. Negative thermal expansion in a zirconium tungstate/epoxy composite at low temperatures. J. Mater. Sci. 2014;49:392-6.

[25] Wang YC, Lakes RS. Extreme thermal expansion, piezoelectricity, and other coupled field properties in composites with a negative stiffness phase. J. Appl. Phys. 2001;90:6458. [26] Alderson A, Alderson KL, Hudson GD, Skertchly DE. A composite material. In:

SPECIFICATION EP, editor. Google Patents 2009.

[27] Pasternak E, Dyskin AV. Materials and structures with macroscopic negative Poisson's ratio. Int. J. Eng. Sci. 2012;52:103-14.

[28] Grima JN, Ellul B, Gatt R, Attard D. Negative thermal expansion from disc, cylindrical, and needle shaped inclusions. Phys. Status Solidi B. 2013;250:2051-6.

[29] Lim T-C. Coefficient of thermal expansion of stacked auxetic and negative thermal expansion laminates. Phys. Status Solidi B. 2011;248:140-7.

[30] Lakes R. Foam structures with a negative poisson's ratio. Science. 1987;235:1038-40.

[31] Smith CW, Grima JN, Evans KE. Novel mechanism for generating auxetic behaviour in reticulated foams: Missing rib foam model. Acta Mater. 2000;48:4349-56.

[32] Alderson K, Alderson A, Ravirala N, Simkins V, Davies P. Manufacture and characterisation of thin flat and curved auxetic foam sheets. Phys. Status Solidi B.

2012;249:1315-21.

[33] Pozniak AA, Smardzewski J, Wojciechowski KW. Computer simulations of auxetic foams in two dimensions. Smart Mater. Struct. 2013;22:084009.

[34] Wojciechowski KW, Brańka AC. Negative Poisson ratio in a two-dimensional "isotropic" solid. Phys. Rev. A. 1989;40:7222-5.

[35] Tretiakov KV. Negative Poisson's ratio of two-dimensional hard cyclic tetramers. J. Non. Cryst. Solids. 2009;355:1435-8.

[36] Narojczyk JW, Wojciechowski KW. Elastic properties of degenerate f.c.c. crystal of polydisperse soft dimers at zero temperature. J. Non·Cryst. Solids. 2010;356:2026-32.

[37] Grima JN, Attard D. Molecular networks with a near zero Poisson's ratio. Phys. Status Solidi B. 2011;248:111-6.

[38] Tretiakov KV, Wojciechowski KW. Elastic properties of fcc crystals of polydisperse soft spheres. Phys. Status Solidi B. 2013:1-10.

[39] Yeganeh-Haeri A, Weidner DJ, Parise JB. Elasticity of $\alpha$-cristobalite: A silicon dioxide with a negative poisson's ratio. Science. 1992;257:650-2.

[40] Baughman RH, Shacklette JM, Zakhidov AA, Stafström S. Negative poisson's ratios as a common feature of cubic metals. Nature. 1998;392:362-5.

[41] Lethbridge ZAD, Williams JJ, Walton RI, Smith CW, Hooper RM, Evans KE. Direct, static measurement of single-crystal Young's moduli of the zeolite natrolite: Comparison with dynamic studies and simulations. Acta Mater. 2006;54:2533-45.

[42] Hall LJ, Coluci VR, Galvão DS, Kozlov ME, Zhang M, Dantas SO, et al. Sign Change of Poisson's Ratio for Carbon Nanotube Sheets. Science. 2008;320:504-7.

[43] Yao YT, Alderson A, Alderson KL. Can nanotubes display auxetic behaviour? Phys. Status Solidi B. 2008;245:2373-82. 
[44] Grima JN, Winczewski S, Mizzi L, Grech MC, Cauchi R, Gatt R, et al. Tailoring graphene to achieve negative Poisson's ratio properties. Adv Mater. 2015;27:1455-9. [45] Phan-Thien N, Karihaloo BL. Materials with negative Poisson's ratio: A qualitative microstructural model. Journal of Applied Mechanics, Transactions ASME. 1994;61:1001-4. [46] Wei G, Edwards SF. Poisson ratio in composites of auxetics. Phys. Rev. E. 1998;58. [47] Hou X, Hu H, Silberschmidt V. A novel concept to develop composite structures with isotropic negative Poisson's ratio: Effects of random inclusions. Compos. Sci. Technol. 2012;72:1848-54.

[48] Assidi M, Ganghoffer J-F. Composites with auxetic inclusions showing both an auxetic behavior and enhancement of their mechanical properties. Compos. Struct. 2012;94:2373-82. [49] Chen YJ, Scarpa F, Farrow IR, Liu YJ, Leng JS. Composite flexible skin with large negative Poisson's ratio range: numerical and experimental analysis. Smart Mater. Struct. 2013;22:045005.

[50] Grima JN, Cauchi R, Gatt R, Attard D. Honeycomb composites with auxetic out-ofplane characteristics. Compos. Struct. 2013;106:150-9.

[51] Hou X, Hu H, Silberschmidt V. Numerical analysis of composite structure with in-plane isotropic negative Poisson's ratio: Effects of materials properties and geometry features of inclusions. Compos. B. Eng. 2014;58:152-9.

[52] Buckmann T, Stenger N, Kadic M, Kaschke J, Frolich A, Kennerknecht T, et al. Tailored 3D mechanical metamaterials made by dip-in direct-laser-writing optical lithography. Adv Mater. 2012;24:2710-4.

[53] Cabras L, Brun M. Auxetic two-dimensional lattices with Poisson's ratio arbitrarily close to -1. Proc. R. Soc. London. Sec. B. 2014;470:20140538-.

[54] Shufrin I, Pasternak E, Dyskin AV. Negative Poisson's ratio in hollow sphere materials. Int. J. Solids Struct. 2015;54:192-214.

[55] Milton GW. Complete characterization of the macroscopic deformations of periodic unimode metamaterials of rigid bars and pivots. J. Mech. Phys. Solids. 2013;61:1543-60.

[56] Grima JN, Caruana-Gauci R, Dudek MR, Wojciechowski KW, Gatt R. Smart metamaterials with tunable auxetic and other properties. Smart Mater. Struct.

2013;22:084016.

[57] Lee JH, Singer JP, Thomas EL. Micro-/nanostructured mechanical metamaterials. Adv Mater. 2012;24:4782-810.

[58] Babaee S, Shim J, Weaver JC, Chen ER, Patel N, Bertoldi K. 3D soft metamaterials with negative Poisson's ratio. Adv Mater. 2013;25:5044-9.

[59] Bertoldi K, Reis PM, Willshaw S, Mullin T. Negative Poisson's Ratio Behavior Induced by an Elastic Instability. Adv. Mater. 2010;22:361-6.

[60] Evans KE, Alderson A. Auxetic Materials: Functional Materials and Structures from Lateral Thinking! Adv. Mater. 2000;12:617-28.

[61] Alderson A, Alderson KL. Auxetic materials. Proc. IME, G, J. Aerospace Eng. 2007;221:565-75.

[62] Prawoto Y. Seeing auxetic materials from the mechanics point of view: A structural review on the negative Poisson's ratio. Comput. Mater. Sci. 2012;58:140-53.

[63] Ostoja-Starzewski M. Material spatial randomness: From statistical to representative volume element. Probab. Eng. Mech. 2006;21:112-32.

[64] MATLAB and Statistics Toolbox Release R2011b. Natick, Massachusetts, United States: The MathWorks Inc.; 2011.

[65] Turner PS. Thermal-Expansion Stresses in Reinforced Plastics. J. Res. Nat. Bur. Stand. 1946;37:239-50.

[66] Levin VM. On the Coefficients of Thermal Expansion of Heterogeneous Materials. Mekhanika Tverdogo Tela (in Russian). 1967;2:58-61. 
[67] Schapery RA. Thermal Expansion Coefficients of Composite Materials Based on Energy Principles. J. Compos. Mater. 1968;2:380-404.

[68] Rosen BW, Hashin Z. Effective thermal expansion coefficients and specific heats of composite materials. Int. J. Eng. Sci. 1970;8:157-73.

[69] Hashin Z. Analysis of Composite Materials - A Survey. J. appl. Mech. 1983;50:481-502. [70] Gubiansky LV, Torquato S. Thermal expansion of isotropic multiphase composites and polycrystals. J. Mech. Phys. Solids. 1997;45:1223-52.

[71] Sevostianov I. On the thermal expansion of composite materials and cross-property connection between thermal expansion and thermal conductivity. Mech. Mater. 2012;45:2033.

[72] Torquato S. Random Heterogeneous Materials: Microstructure and Macroscopic Properties: Springer, 2002.

[73] Hashin Z, Shtrikman S. A variational approach to the theory of the elastic behaviour of multiphase materials. J. Mech. Phys. Solids. 1963;11:127-40. 


\section{Appendix A: Evaluation of effective bulk modulus for a hybrid with randomly distributed cubic inclusions with negative Poisson's ratio}

In order to determine the bulk modulus of the hybrid with cubic auxetic inclusions, we simulate the uniform compression test in ABAQUS, a finite element commercial package [2]. We find the bulk modulus using a cubic Representative Volume Element (RVE) defined and modelled as described in Section 2. Similarly we assume that the RVE is symmetric in all three directions and reduce the computational model to an eighth of the cubic RVE as shown in Fig A1. Fig A1 also presents the imposed boundary conditions resulting from the symmetry assumption (1) and the boundary conditions at the outer faces of the RVE.

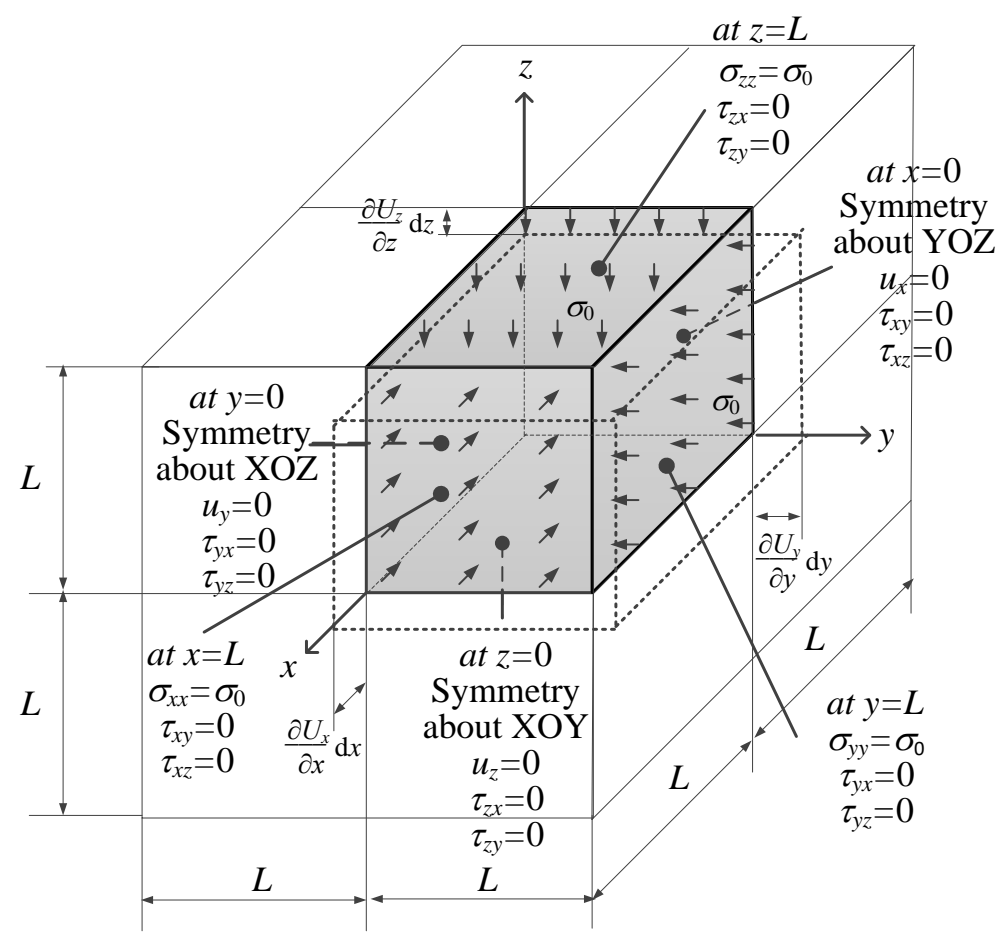

Fig A1: Compressive test computational model: RVE, symmetry considerations, loading and boundary conditions.

The RVE is loaded in all three directions by uniformly distributed pressure $\sigma_{0}$ and the normal displacements are calculated at each face by the finite element package. The generalised strain in every direction is obtained as an average displacement over the corresponding face divided by the size of the RVE. The statistical validation is provided by analysing of 10 random realisations of the distribution of the inclusions for each volume fraction. The bulk modulus of the hybrid is found as 


$$
K_{e f f}=\frac{\sigma_{0}}{\hat{\varepsilon}_{x x}+\hat{\varepsilon}_{y y}+\hat{\varepsilon}_{z z}}
$$

where $\sigma_{0}$ is the applied stress (see Fig. A1), $\hat{\varepsilon}_{x x}, \hat{\varepsilon}_{y y}$ and $\hat{\varepsilon}_{z z}$ are the mean generalised strains obtained by averaging the strains over the realisations. 


\section{List of Figures and Tables}

Fig 1: Representative volume element for the hybrid material with randomly distributed auxetic cubic inclusions. Each cubic inclusion is modelled by a number of 3D finite elements.

Fig 2: Free thermal expansion test: RVE geometry, symmetry and boundary conditions.

Fig 3: Confined thermal loading test: RVE geometry, symmetry and boundary conditions.

Fig 4: Comparisons of the effective CTE for the hybrids with auxetic inclusions calculated using different methods: $v_{i n}=-0.90, v_{m}=0.45, E_{i n} / E_{m}=1, \alpha_{i n} / \alpha_{m}=-10$. The error bars of standard deviations calculated based on 10 realisations of inclusion distributions are not visible since the error is less than the symbol size

Fig 5: Effective Young's and bulk moduli for the hybrid with auxetic inclusions and negative thermal expansion calculated via the tensile test: $v_{i n}=-0.9, v_{m}=0.45, E_{i n} / E_{m}=1$. The error bars of standard deviations calculated based on 10 realisations of inclusion distributions are not visible since the error is less than the symbol size

Fig 6: The effective CTE for the hybrids with the negative CTE inclusions with various Poisson's ratios, $v_{i n}=-0.99 \ldots 0.49, v_{m}=0.45, E_{i n} / E_{m}=1, \alpha_{i n} / \alpha_{m}=-10$. The error bars of standard deviations calculated based on 10 realisations of inclusion distributions are not visible since the error is less than the symbol size

Fig 7: The effective CTE for the hybrids with the positive CTE inclusions with various levels of Poisson's ratio, $v_{i n}=-0.99 \ldots 0.49, v_{m}=0.45, E_{i n} / E_{m}=1, \alpha_{i n} / \alpha_{m}=10$. The error bars of standard deviations calculated based on 10 realisations of inclusion distributions are not visible since the error is less than the symbol size

Fig 8: The effective CTE for the hybrids with the auxetic inclusions with various Young's moduli, $v_{i n}=-0.99, v_{m}=0.45, E_{i n} / E_{m}=0.0001 \ldots 1, \alpha_{i n} / \alpha_{m}=0.1$. The error bars of standard deviations calculated based on 10 realisations of inclusion distributions are not visible since the error is less than the symbol size

Fig 9: Thermal stresses $\sigma_{x} / \sigma_{x m}$ in the hybrids with the negative CTE inclusions with negative and positive magnitudes of Poisson's ratio, $v_{m}=0.45, E_{i n} / E_{m}=1, \alpha_{i n} / \alpha_{m}=-10$, a) $v_{i n}=-$ $0.99 \ldots 0.2, b) v_{i n}=0.2 \ldots 0.49$. The error bars of standard deviations calculated based on 10 realisations of inclusion distributions are not visible since the error is less than the symbol size

Fig 10: Thermal stresses $\sigma_{x} / \sigma_{x m}$ in the hybrids with the auxetic (a) and conventional (b) inclusions with various Poisson's ratios and the positive CTE that is smaller than the matrix CTE, $v_{m}=0.45, E_{i n} / E_{m}=1, \alpha_{i n} / \alpha_{m}=0.1$, a) $v_{i n}=-0.99 \ldots 0$, b) $v_{i n}=0 \ldots 0.49$. The error bars of standard deviations calculated based on 10 realisations of inclusion distributions are not visible since the error is less than the symbol size. The error bars of standard deviations calculated based on 10 realisations of inclusion distributions are not visible since the error is less than the symbol size. 
Fig 11: Thermal stresses $\sigma_{x} / \sigma_{x m}$ in the hybrids with the auxetic (a) and conventional (b) inclusions with various Poisson's ratios and the positive CTE that is higher than the matrix CTE, $v_{m}=0.45, E_{i n} / E_{m}=1, \alpha_{i n} / \alpha_{m}=10$, a) $v_{i n}=-0.99 \ldots-0.4$, b) $v_{i n}=-0.4 \ldots 0.49$. The error bars of standard deviations calculated based on 10 realisations of inclusion distributions are not visible since the error is less than the symbol size

Fig 12: Thermal stresses $\sigma_{x} / \sigma_{x m}$ in the hybrids with the auxetic inclusions of various coefficients of thermal expansion, $v_{i n}=-0.9, v_{m}=0.45, E_{i n} / E_{m}=1, \alpha_{i n} / \alpha_{m}=-10 \ldots 10$. The error bars of standard deviations calculated based on 10 realisations of inclusion distributions are not visible since the error is less than the symbol size

Fig 13: Thermal stresses $\sigma_{x} / \sigma_{x m}$ in the hybrids with the auxetic inclusions with various Young's moduli, $v_{i n}=-0.99, v_{m}=0.45, E_{i n} / E_{m}=0.0001 \ldots 1, \alpha_{i n} / \alpha_{m}=0.1$. The error bars of standard deviations calculated based on 10 realisations of inclusion distributions are not visible since the error is less than the symbol size

Table 1: Comparisons of the effective CTE for the composites with NTE phase: zirconium tungstate particles with a cyanate ester resin matrix

Table 2: Comparisons of the effective CTE for the composites with NTE phase: zirconium tungstate/epoxy particulate composite 


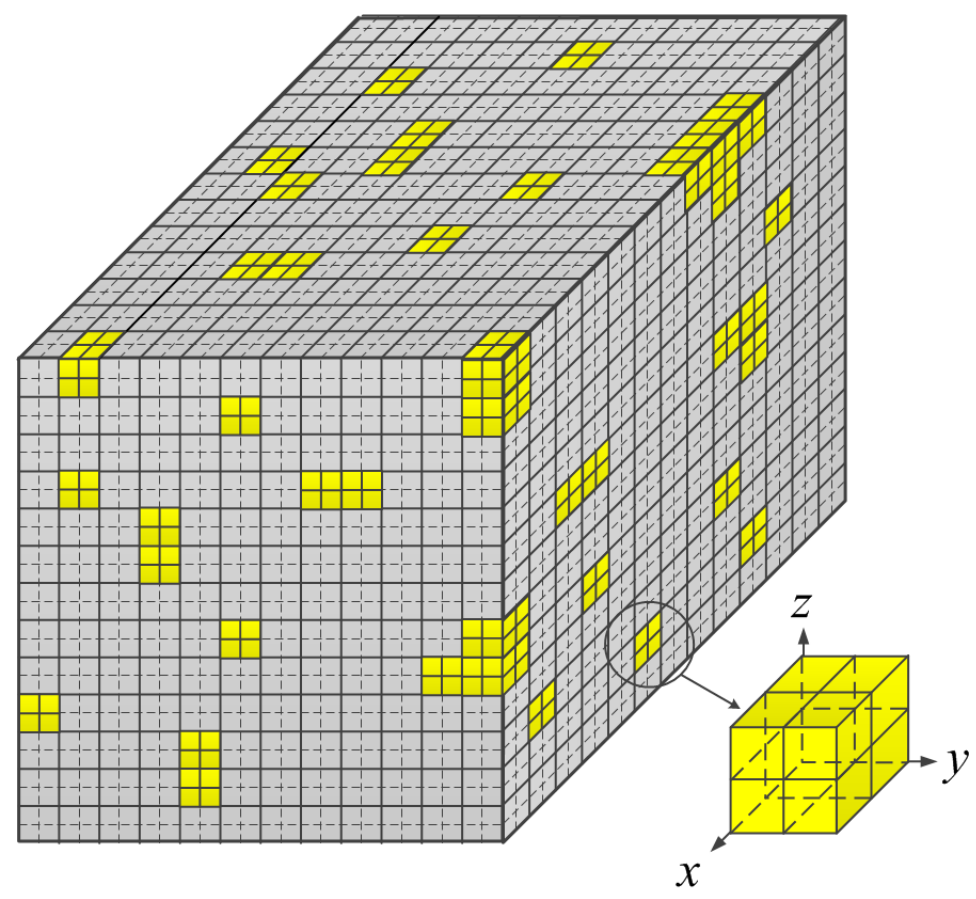

Fig 1: Representative volume element for the hybrid material with randomly distributed auxetic cubic inclusions. Each cubic inclusion is modelled by a number of 3D finite elements.

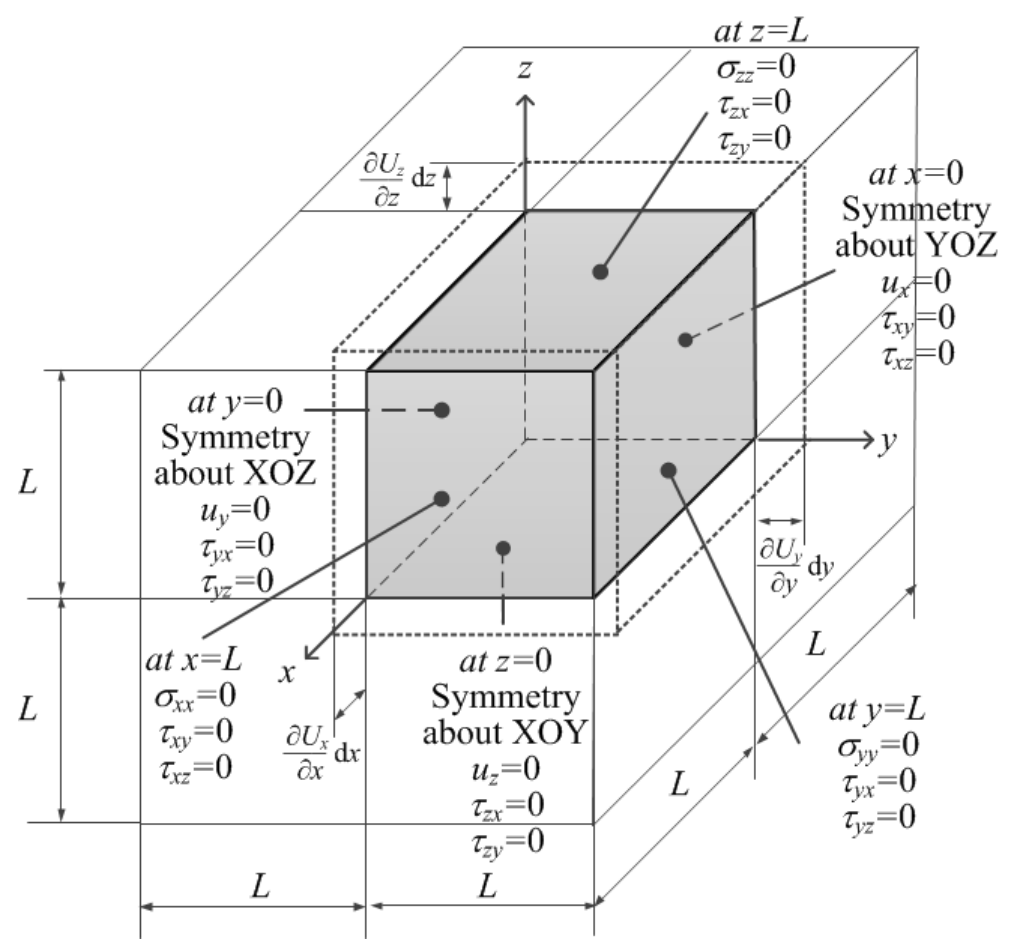

Fig 2: Free thermal expansion test: RVE geometry, symmetry and boundary conditions. 


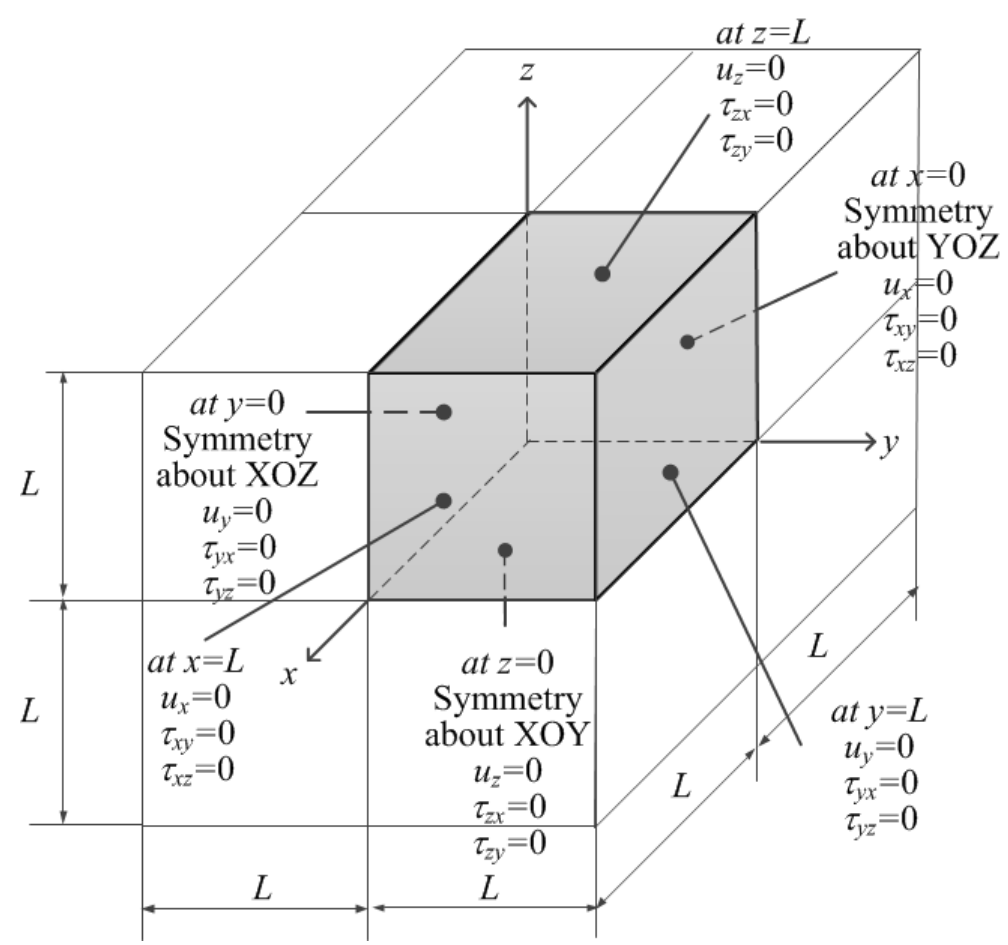

Fig 3: Confined thermal loading test: RVE geometry, symmetry and boundary conditions.

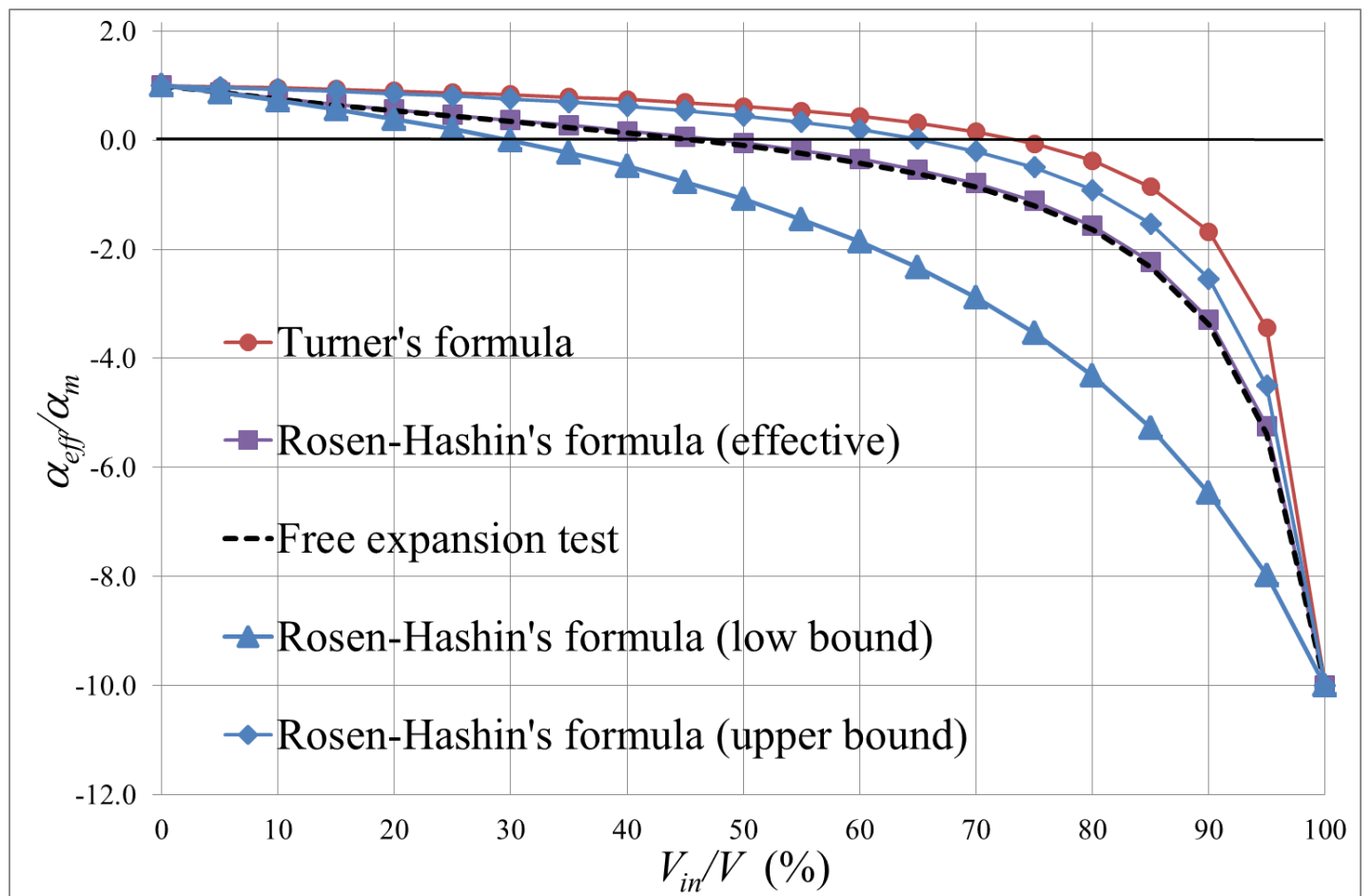

Fig 4: Comparisons of the effective CTE for the hybrids with auxetic inclusions calculated using different methods: $v_{i n}=-0.90, v_{m}=0.45, E_{i n} / E_{m}=1, \alpha_{i n} / \alpha_{m}=-10$. The error bars of standard deviations calculated based on 10 realisations of inclusion distributions are not visible since the error is less than the symbol size. 


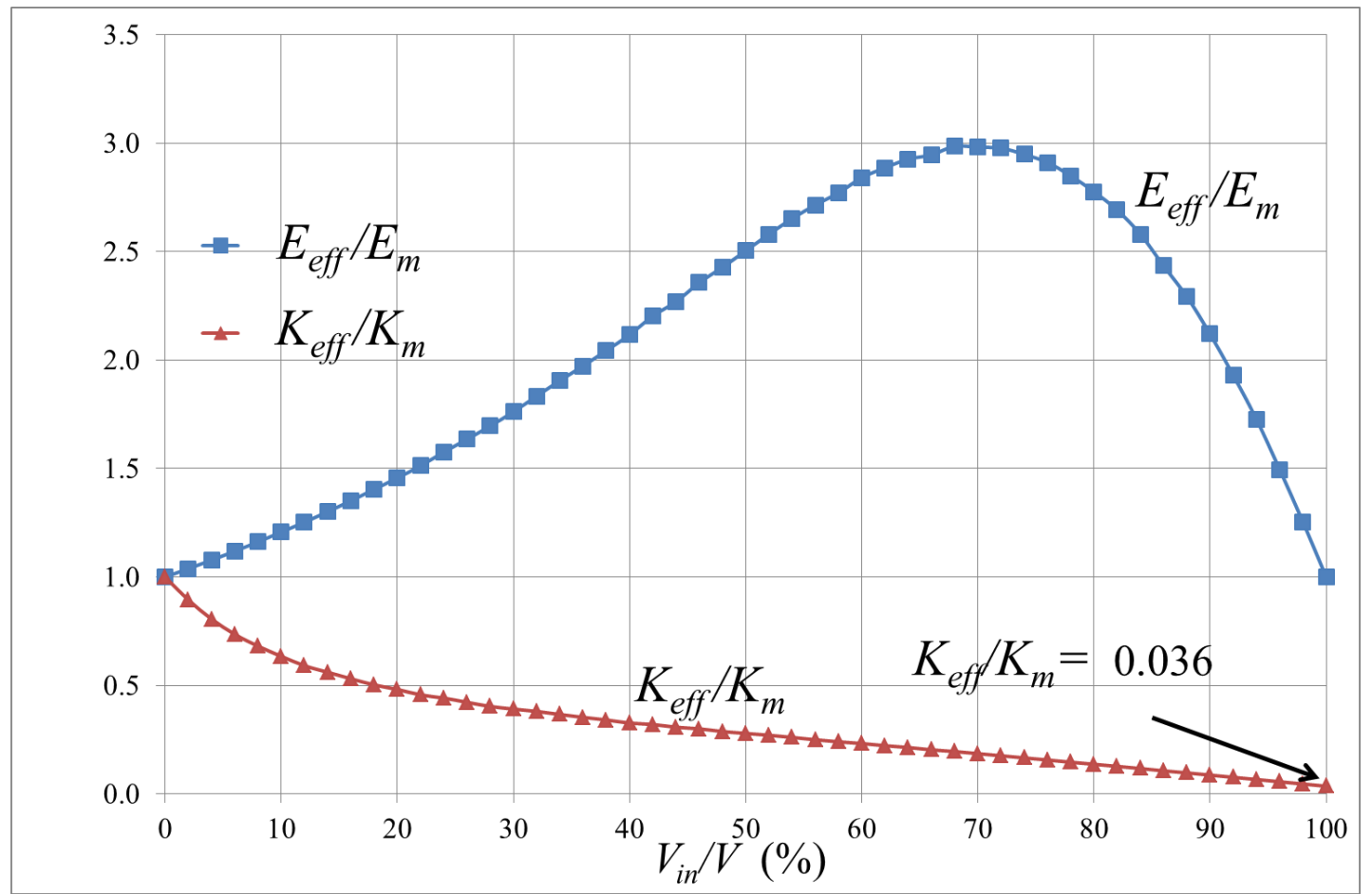

Fig 5: Effective Young's and bulk moduli for the hybrid with auxetic inclusions and negative thermal expansion calculated via the tensile test: $v_{i n}=-0.9, v_{m}=0.45, E_{i n} / E_{m}=1$. The error bars of standard deviations calculated based on 10 realisations of inclusion distributions are not visible since the error is less than the symbol size.

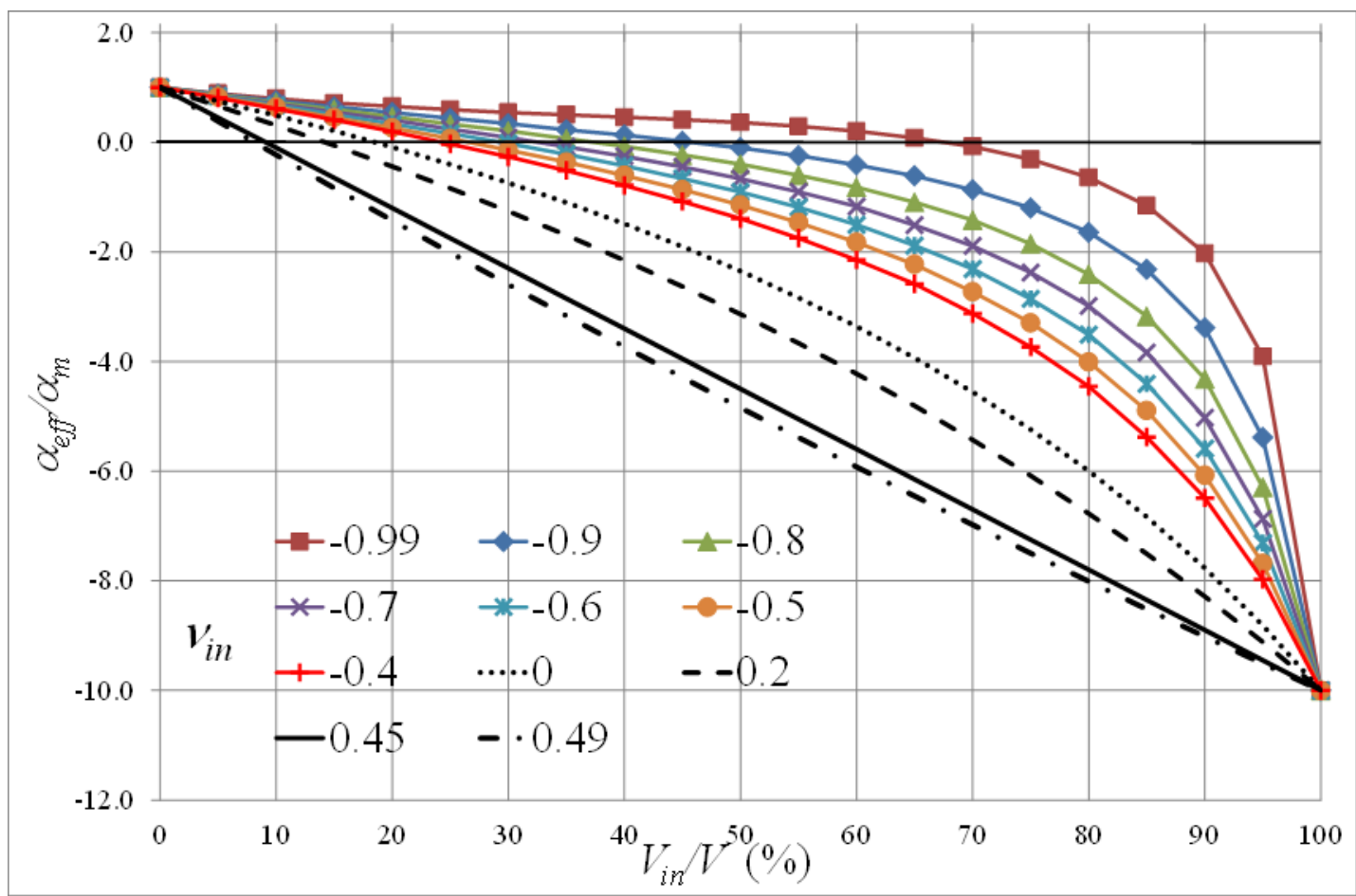

Fig 6: The effective CTE for the hybrids with the negative CTE inclusions with various Poisson's ratios, $v_{i n}=-0.99 \ldots 0.49, v_{m}=0.45, E_{i n} / E_{m}=1, \alpha_{i n} / \alpha_{m}=-10$. The error bars of standard deviations calculated based on 10 realisations of inclusion distributions are not visible since the error is less than the symbol size 


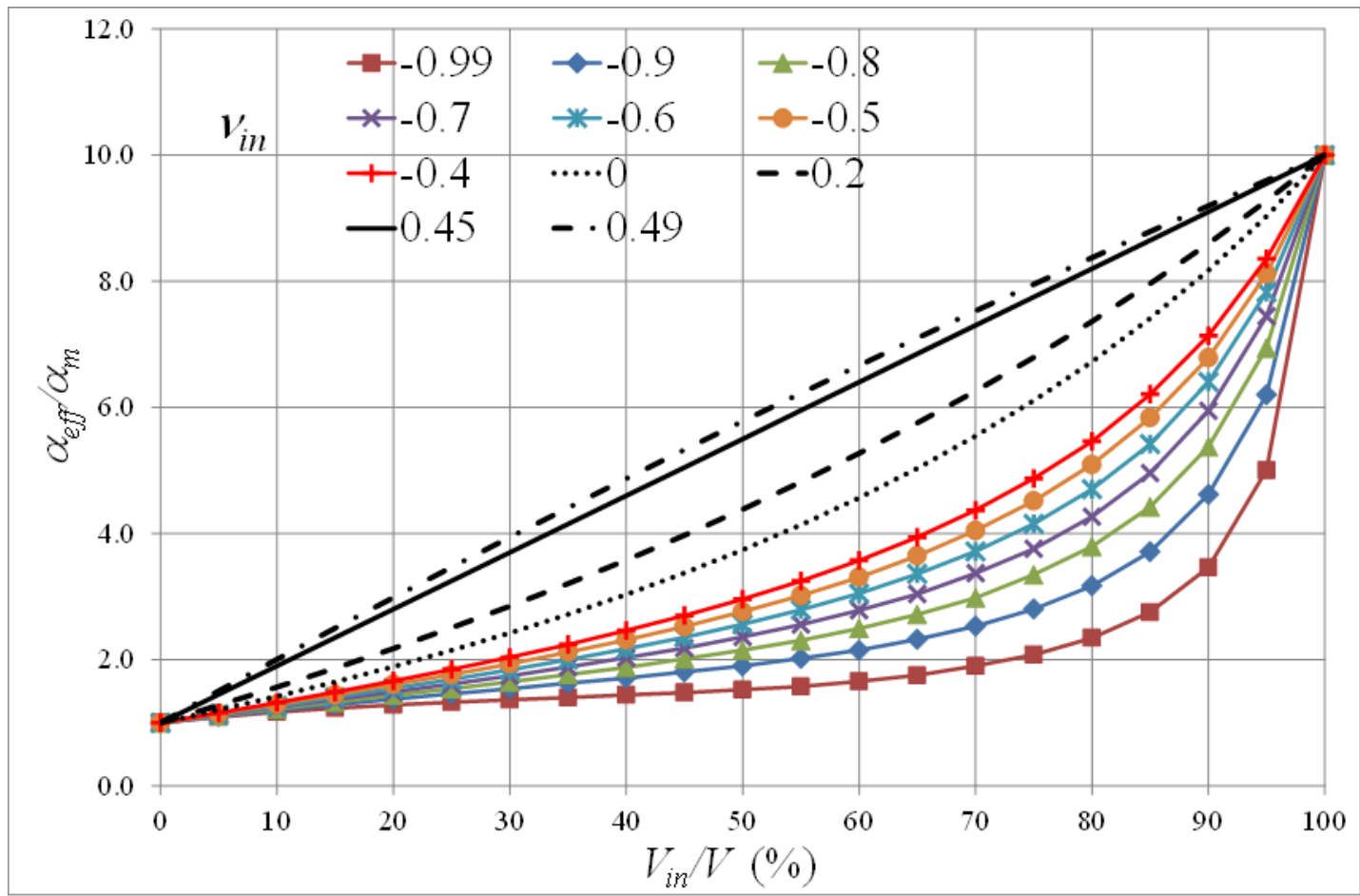

Fig 7: The effective CTE for the hybrids with the positive CTE inclusions with various levels of Poisson's ratio, $v_{i n}=-0.99 \ldots 0.49, v_{m}=0.45, E_{i n} / E_{m}=1, \alpha_{i n} / \alpha_{m}=10$. The error bars of standard deviations calculated based on 10 realisations of inclusion distributions are not visible since the error is less than the symbol size

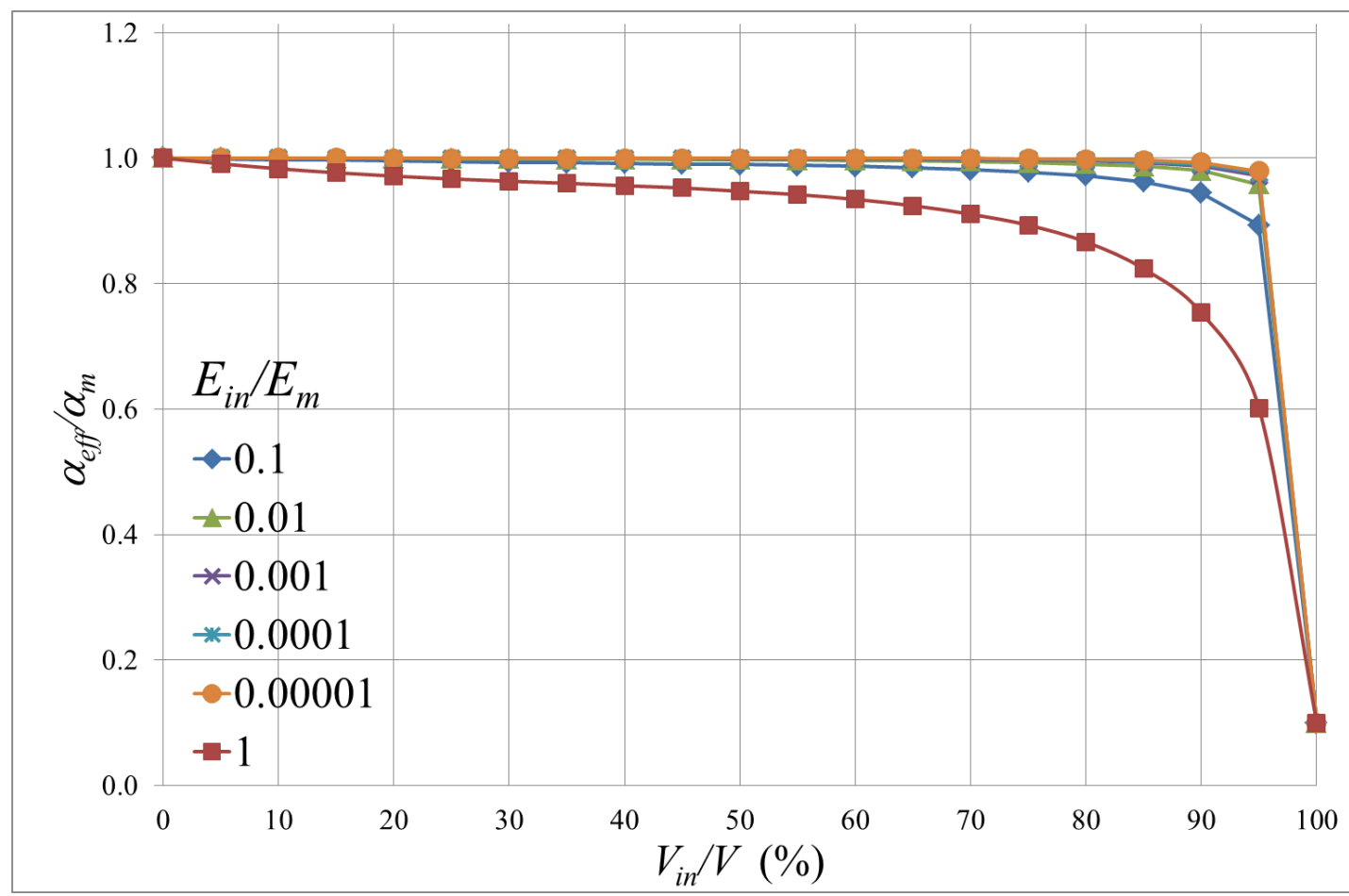

Fig 8: The effective CTE for the hybrids with the auxetic inclusions with various Young's moduli, $v_{i n}=-0.99, v_{m}=0.45, E_{i n} / E_{m}=0.0001 \ldots 1, \alpha_{i n} / \alpha_{m}=0.1$. The error bars of standard deviations calculated based on 10 realisations of inclusion distributions are not visible since the error is less than the symbol size. 


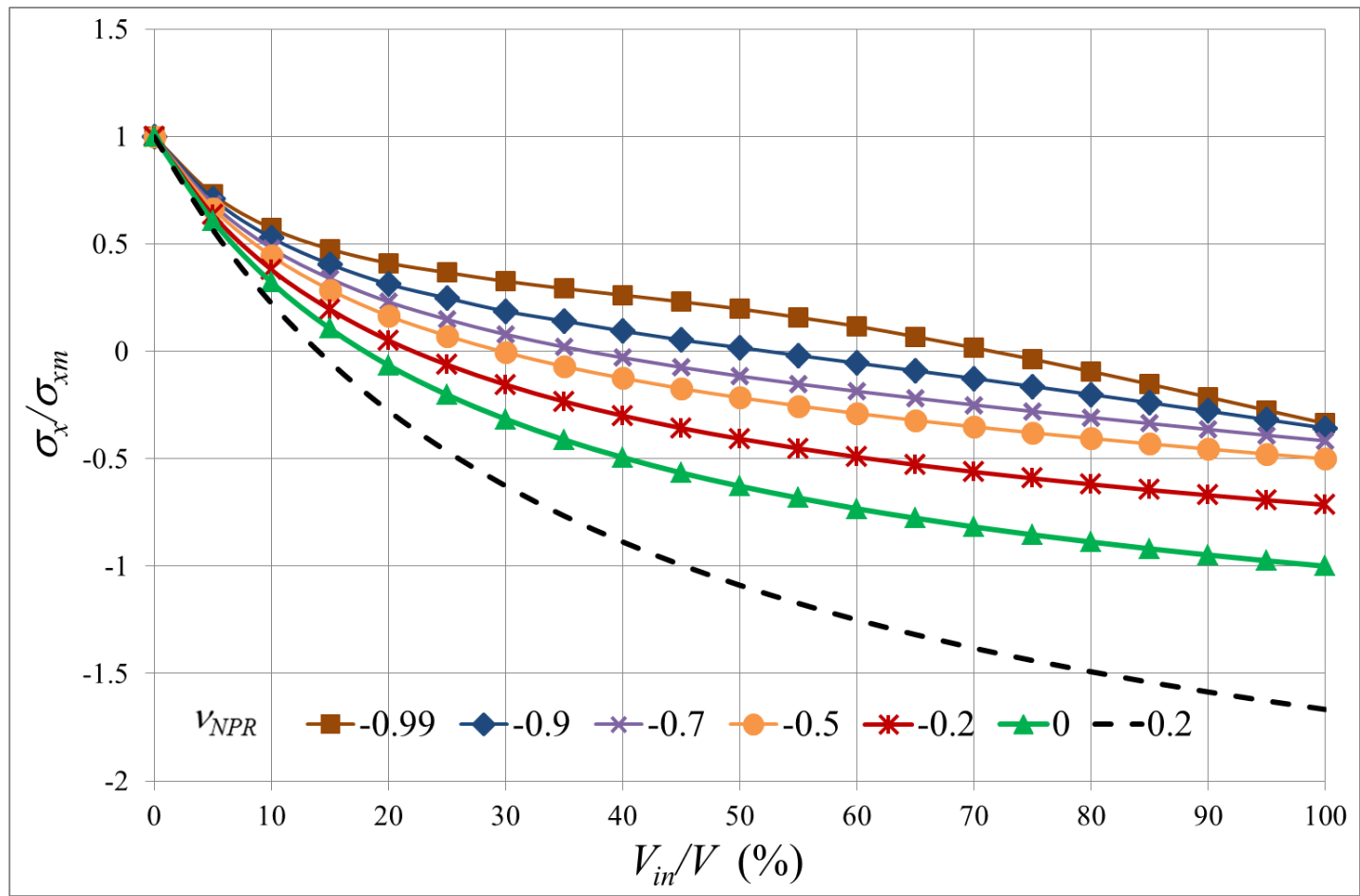

(a)

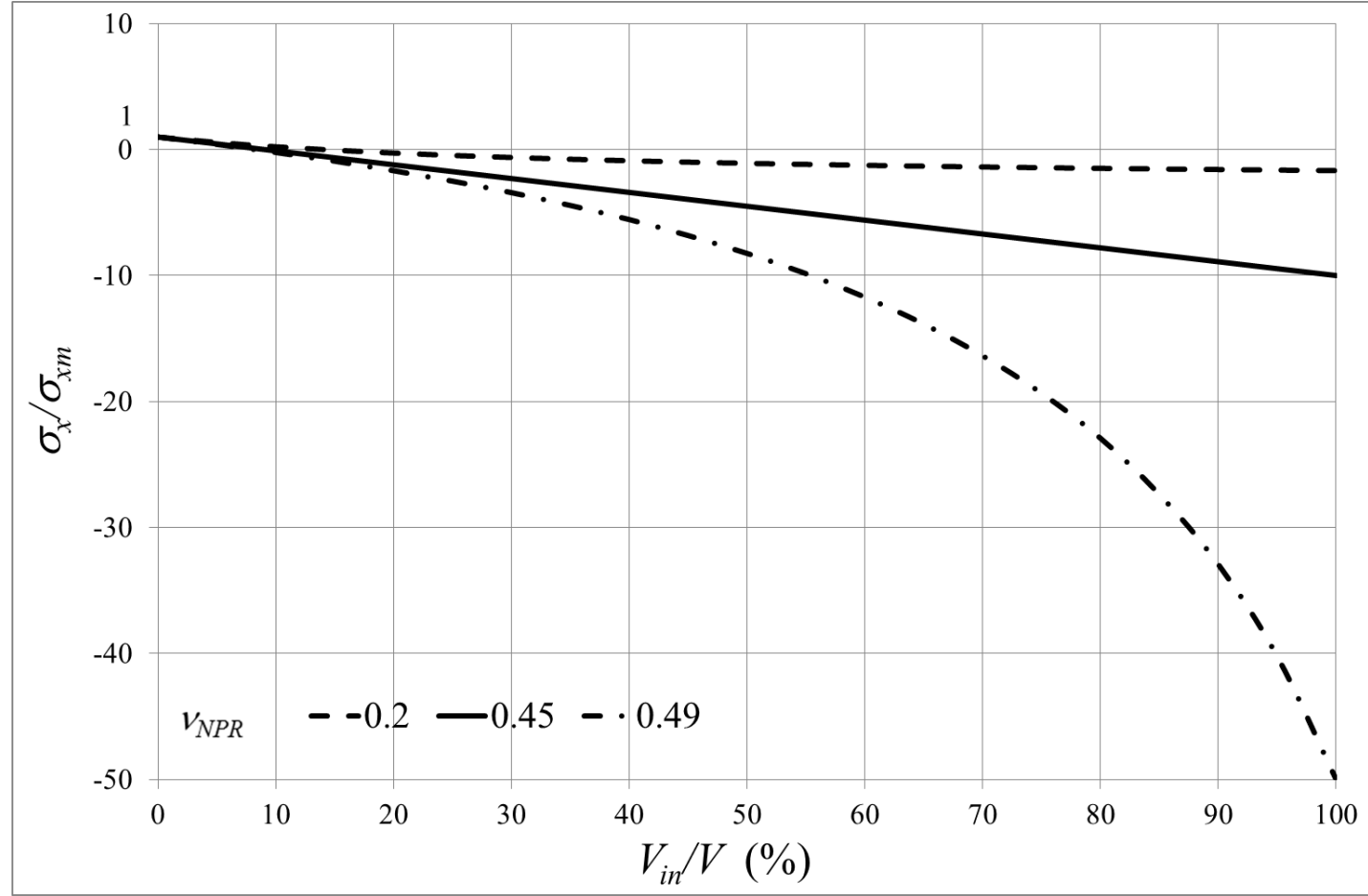

(b)

Fig 9: Thermal stresses $\sigma_{x} / \sigma_{x m}$ in the hybrids with the negative CTE inclusions with negative and positive magnitudes of Poisson's ratio, $v_{m}=0.45, E_{i n} / E_{m}=1, \alpha_{i n} / \alpha_{m}=-10$, a) $v_{i n}=-$ $0.99 \ldots 0.2, b) v_{i n}=0.2 \ldots 0.49$. The error bars of standard deviations calculated based on 10 realisations of inclusion distributions are not visible since the error is less than the symbol size. 


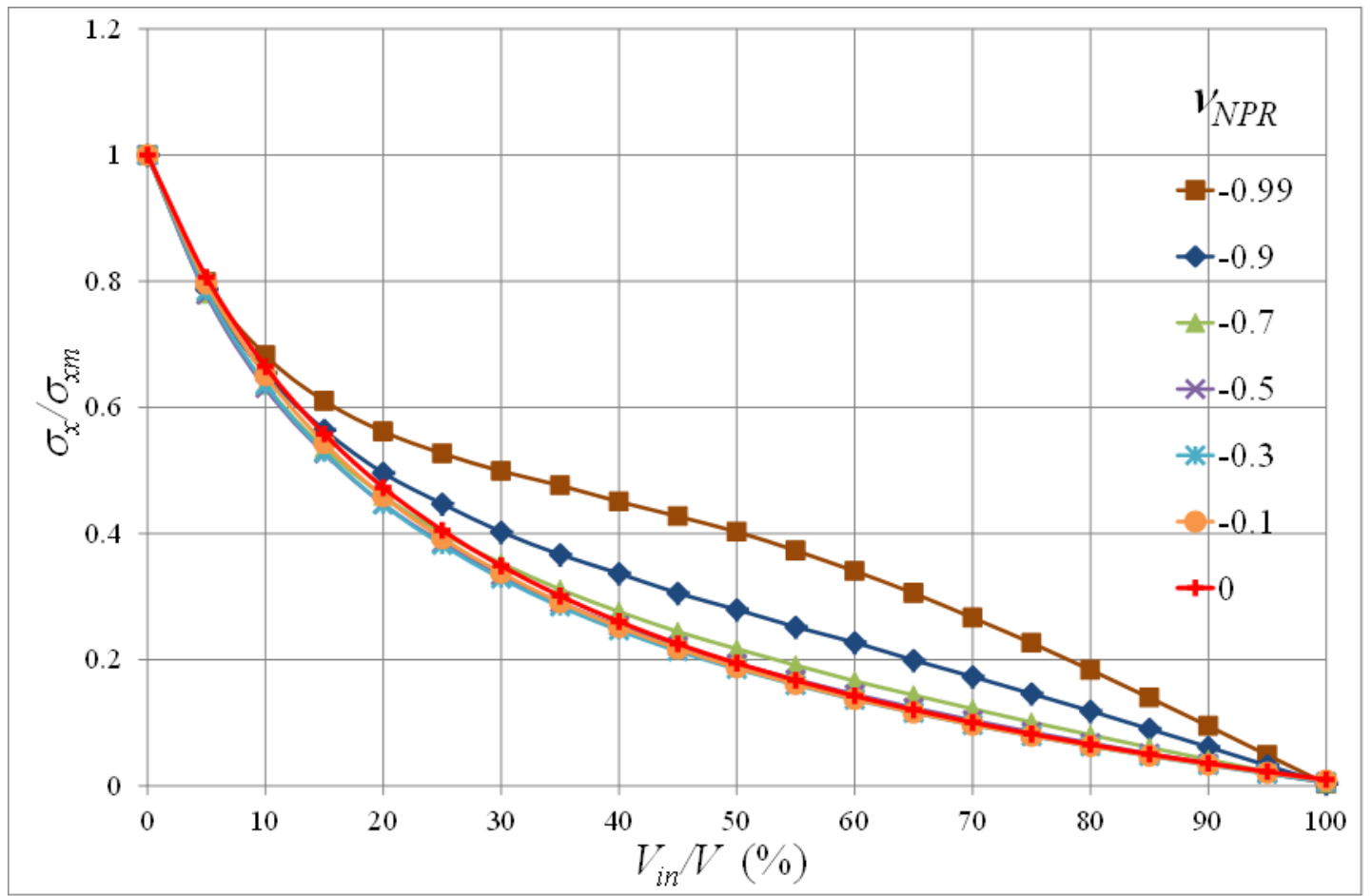

(a)

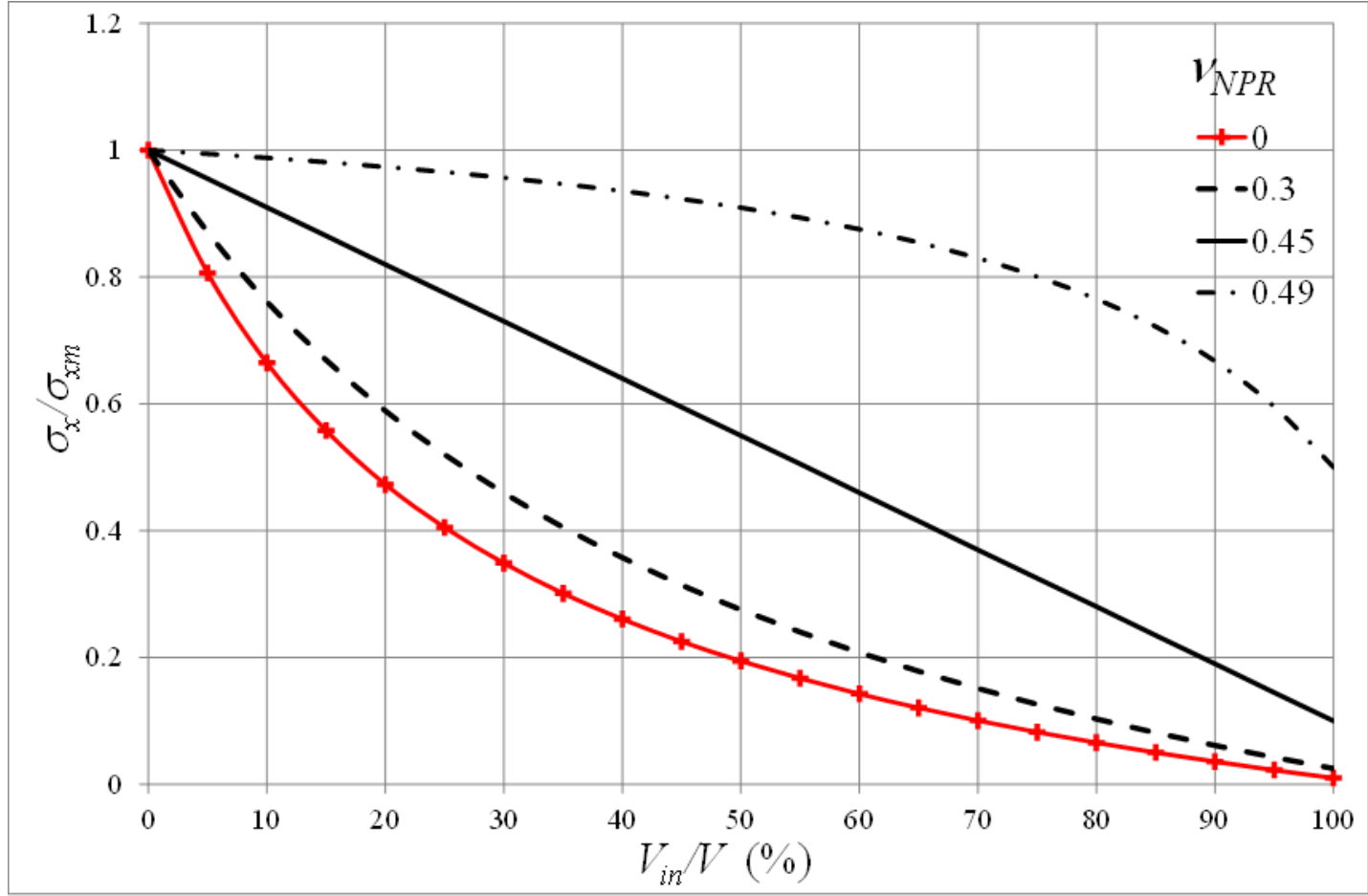

(b)

Fig 10: Thermal stresses $\sigma_{x} / \sigma_{x m}$ in the hybrids with the auxetic (a) and conventional (b) inclusions with various Poisson's ratios and the positive CTE that is smaller than the matrix CTE, $v_{m}=0.45, E_{i n} / E_{m}=1, \alpha_{i n} / \alpha_{m}=0.1$, a) $v_{i n}=-0.99 \ldots 0$, b) $v_{i n}=0 \ldots 0.49$. The error bars of standard deviations calculated based on 10 realisations of inclusion distributions are not visible since the error is less than the symbol size 


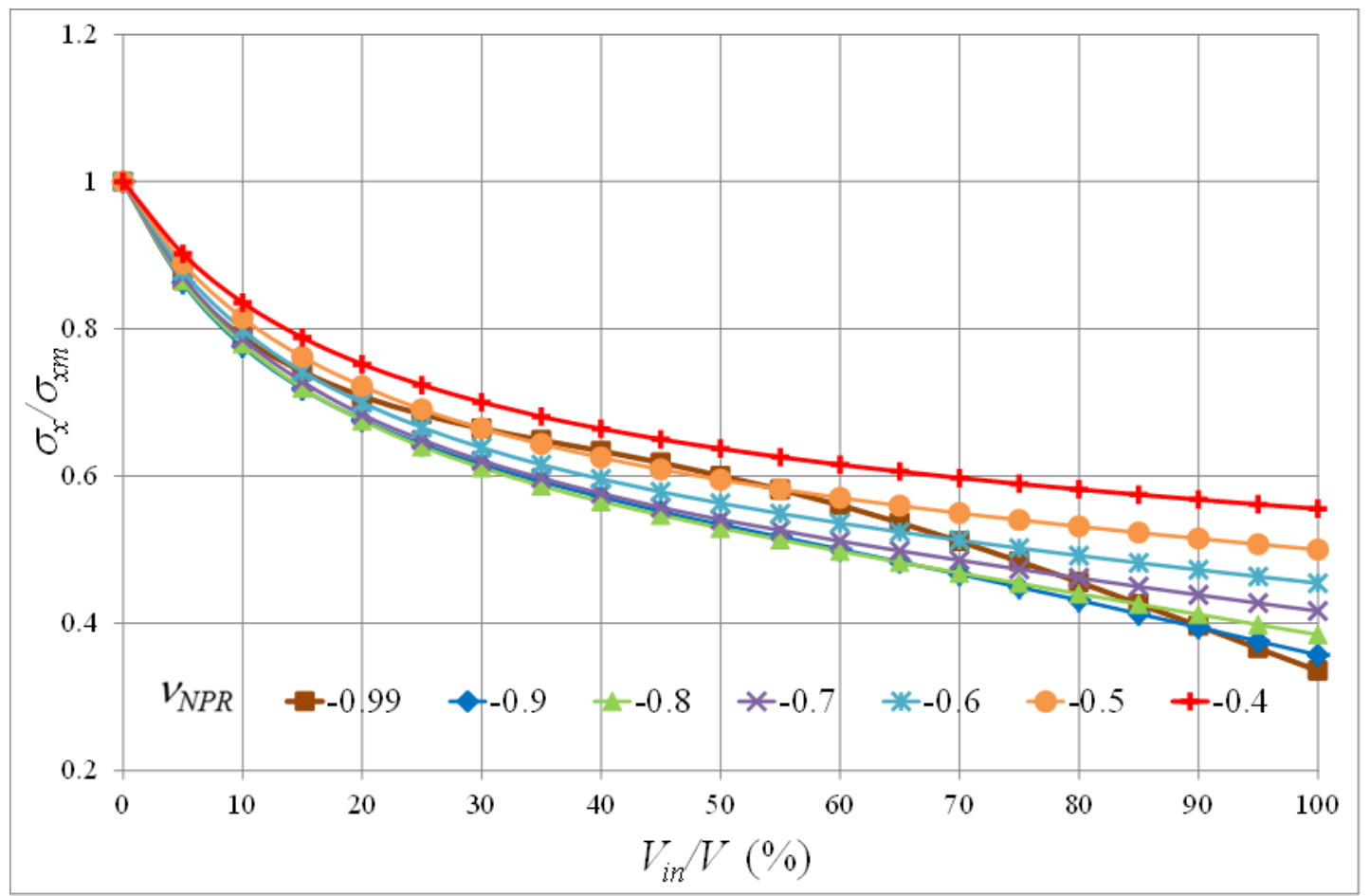

(a)

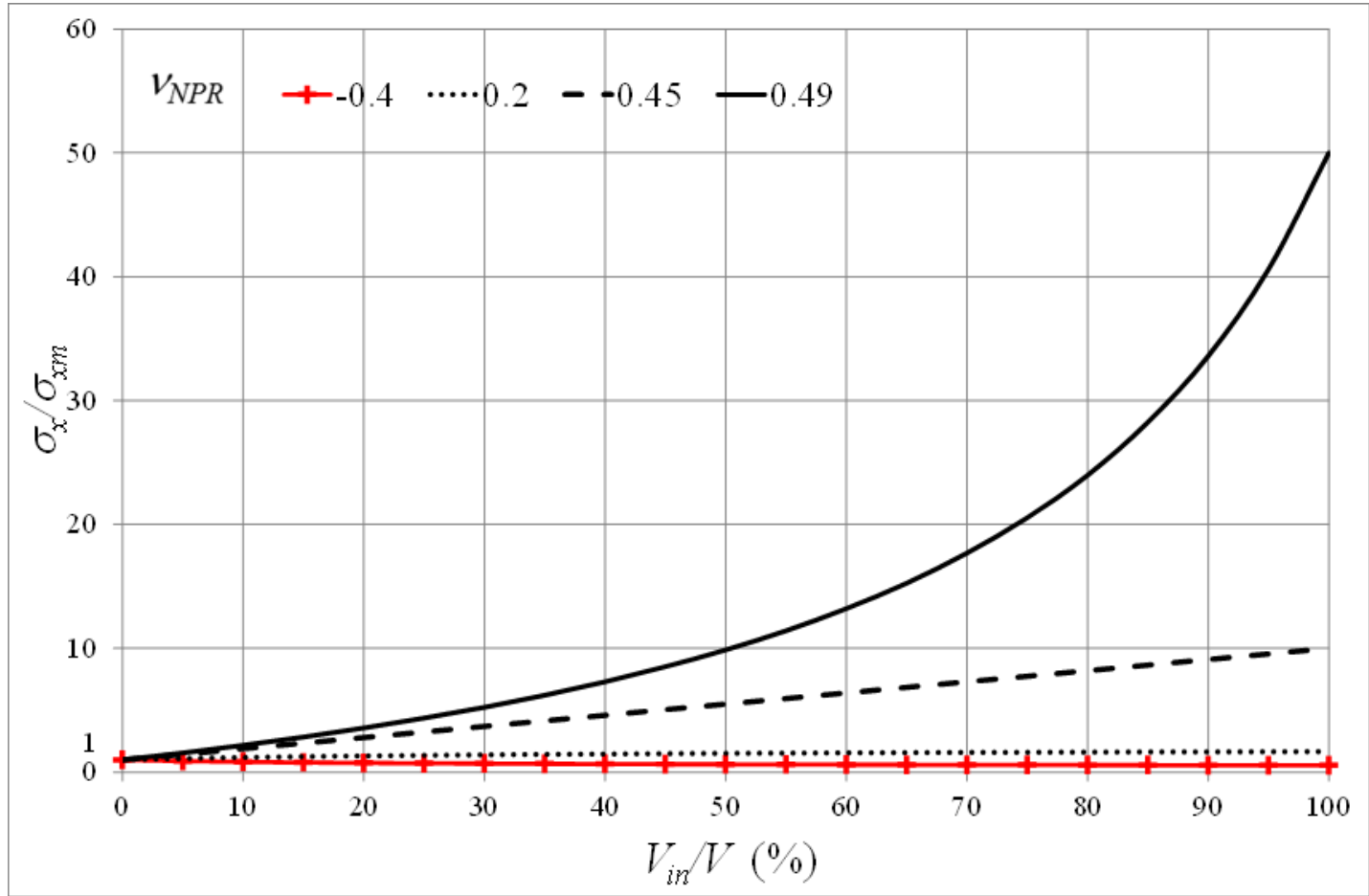

(b)

Fig 11: Thermal stresses $\sigma_{x} / \sigma_{x m}$ in the hybrids with the auxetic (a) and conventional (b) inclusions with various Poisson's ratios and the positive CTE that is higher than the matrix CTE, $v_{m}=0.45, E_{i n} / E_{m}=1, \alpha_{i n} / \alpha_{m}=10$, a) $v_{i n}=-0.99 \ldots-0.4$, b) $v_{i n}=-0.4 \ldots 0.49$. The error bars of standard deviations calculated based on 10 realisations of inclusion distributions are not visible since the error is less than the symbol size 


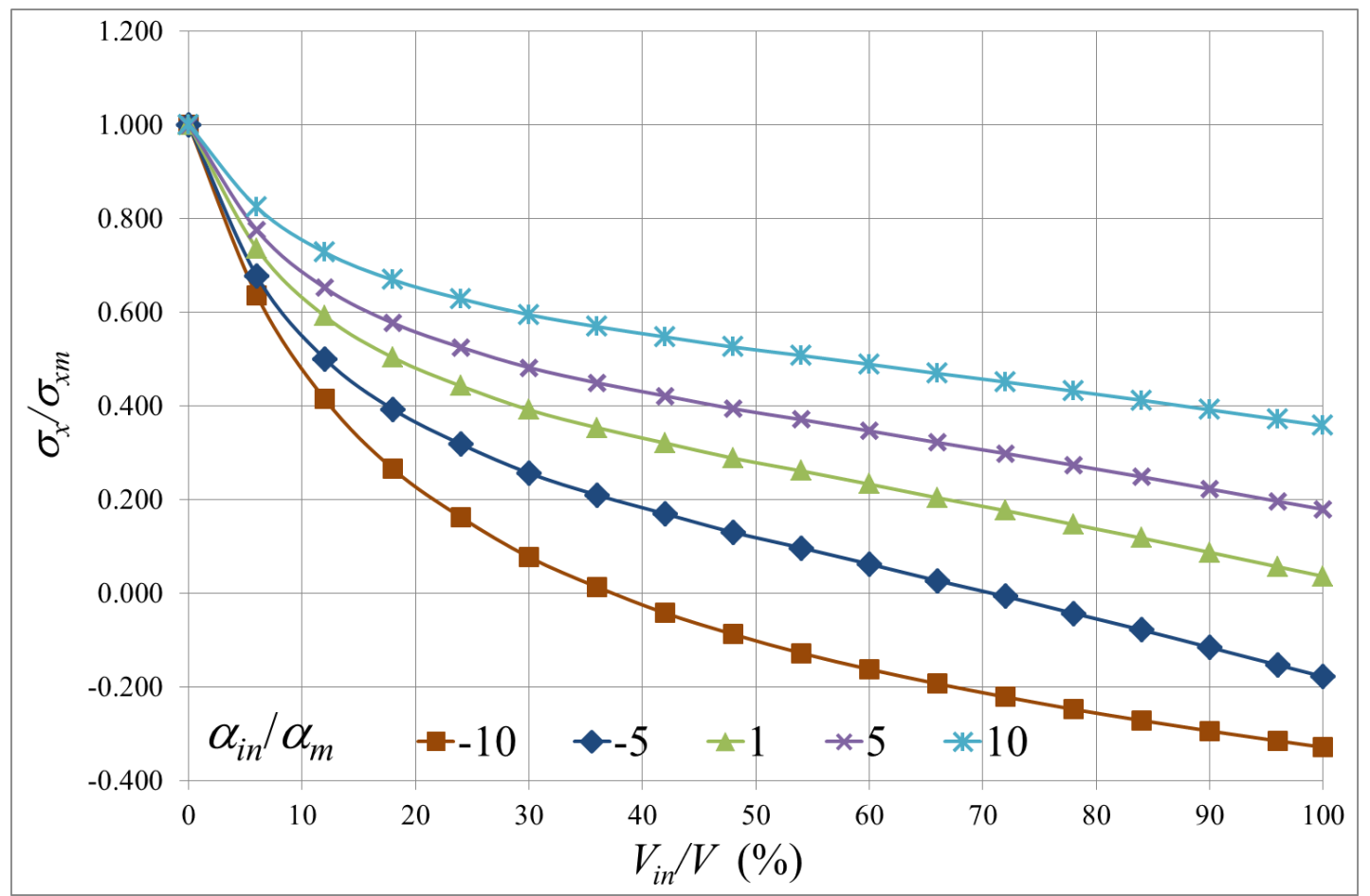

Fig 12: Thermal stresses $\sigma_{x} / \sigma_{x m}$ in the hybrids with the auxetic inclusions of various coefficients of thermal expansion, $v_{i n}=-0.9, v_{m}=0.45, E_{i n} / E_{m}=1, \alpha_{i n} / \alpha_{m}=-10 \ldots 10$. The error bars of standard deviations calculated based on 10 realisations of inclusion distributions are not visible since the error is less than the symbol size

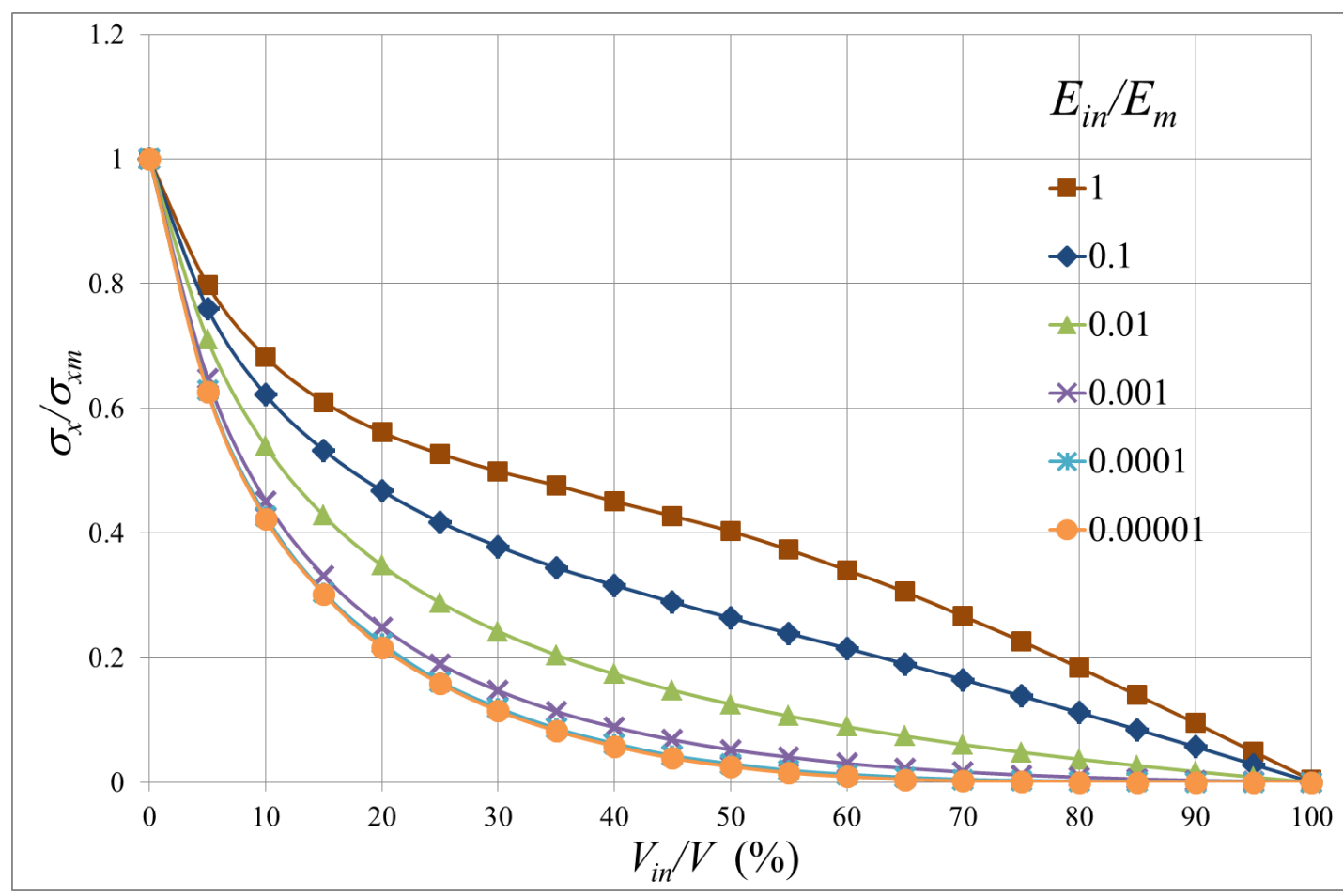

Fig 13: Thermal stresses $\sigma_{x} / \sigma_{x m}$ in the hybrids with the auxetic inclusions with various Young's moduli, $v_{i n}=-0.99, v_{m}=0.45, E_{i n} / E_{m}=0.0001 \ldots 1, \alpha_{i n} / \alpha_{m}=0.1$. The error bars of standard deviations calculated based on 10 realisations of inclusion distributions are not visible since the error is less than the symbol size 
Table 1: Comparisons of the effective CTE for the composites with NTE phase: zirconium tungstate particles with a cyanate ester resin matrix.

\begin{tabular}{ccccc}
\hline \multirow{2}{*}{$\begin{array}{c}V_{i n} / V \\
\%\end{array}$} & \begin{tabular}{c} 
Experimental \\
\cline { 2 - 5 }
\end{tabular} & $\begin{array}{c}\text { Rosen-Hashin (6) } \\
\text { (low bound) }\end{array}$ & $\begin{array}{c}\text { Rosen-Hashin (6) } \\
\text { (upper bound) }\end{array}$ & Present study \\
\hline $0 \%$ & 56 & 56.50 & 56.50 & 56.5 \\
$1 \%$ & 55 & 48.21 & 55.53 & 55.5 \\
$3 \%$ & 53 & 36.39 & 53.63 & 53.4 \\
$5 \%$ & 50 & 28.37 & 51.76 & 51.3 \\
$10 \%$ & 45 & 16.35 & 47.24 & 45.7 \\
\hline
\end{tabular}

Table 2: Comparisons of the effective CTE for the composites with NTE phase: zirconium tungstate/epoxy particulate composite

\begin{tabular}{|c|c|c|c|c|c|c|}
\hline \multirow[b]{3}{*}{$\begin{array}{c}V_{\text {in }} / V \\
\%\end{array}$} & \multicolumn{6}{|c|}{ Effective CTE, $\times 10^{-6} \mathrm{~K}^{-1}$} \\
\hline & \multicolumn{3}{|c|}{ Experimental [19] } & \multirow[b]{2}{*}{$\begin{array}{c}\text { Rosen- } \\
\text { Hashin (6) } \\
\text { (low bound) }\end{array}$} & \multirow[b]{2}{*}{$\begin{array}{l}\text { Rosen- } \\
\text { Hashin (6) } \\
\text { (upper } \\
\text { bound) }\end{array}$} & \multirow[b]{2}{*}{$\begin{array}{c}\text { Present } \\
\text { study }\end{array}$} \\
\hline & $\begin{array}{l}\text { Uncoated } \\
\text { filler } \\
\text { particles }\end{array}$ & $\begin{array}{c}\text { Silane } \\
\text { surface } \\
\text { coated filler } \\
\text { particles }\end{array}$ & $\begin{array}{l}\text { Carboxylic } \\
\text { acid coated } \\
\text { filler } \\
\text { particles }\end{array}$ & & & \\
\hline $10 \%$ & 190 & 183 & 192 & 57.8 & 168.6 & 162.3 \\
\hline $20 \%$ & 151 & 153 & 161 & 27.2 & 140.6 & 120.8 \\
\hline $30 \%$ & 135 & 164 & 128 & 14.0 & 115.2 & 80.4 \\
\hline $40 \%$ & 144 & 123 & 92 & 6.4 & 92.4 & 48.4 \\
\hline
\end{tabular}

